\title{
Cu(I) Tol-BINAP-Catalyzed Enantioselective Michael Reactions of Grignard reagents and Unsaturated Esters
}

\author{
Shun-Yi Wang, ${ }^{\dagger, \ddagger}$ Shun-Jun Ji, ${ }^{*, \ddagger}$ and Teck-Peng Loh ${ }^{*, \dagger}$ \\ ${ }^{\dagger}$ Division of Chemistry and Biological Chemistry, School of Physical and Mathematical Sciences, Nanyang \\ Technological University, Singapore 639798 \\ ${ }^{\ddagger}$ Key Lab. of Organic Synthesis of Jiangsu Province, College of Chemistry and Chemical Engineering, Suzhou \\ (Soochow) University, Jiangsu 215123, China
}




\section{Supporting Information}

Table of Contents

Page

$\begin{array}{ll}\text { General Methods } & \text { S3 }\end{array}$

Table 1: Additions of EtMgBr to 2: Ligands effect $\quad$ S6

Preparation and characterization of $\mathrm{Cu}$-complexe 1a $\quad$ S8

General Procedure for the Enantioselective Conjugate Addition $\quad$ S9

$\begin{array}{ll}\text { Characterization of CA products } & \mathrm{S} 10\end{array}$

The crystal-structure of $\mathrm{Cu}$ dimer complex 1a $\quad \mathrm{S} 30$ 


\section{General Methods}

Experiments involving moisture and/or air sensitive components were performed in oven-dried glassware under a positive pressure of nitrogen using freshly distilled solvents. Commercial grade solvents and reagents were used without further purification with the following exceptions: $t$-BuOMe was distilled from calcium hydride. Dichloromethane was distilled from calcium hydride. Diethyl Ether was distilled from sodium. Hexane, ethyl acetate were fractionally distilled.

Copper (I) salts (CuI, $\mathrm{CuCl}, \mathrm{CuBr}, \mathrm{CuBr} \mathrm{SMe}_{2} \mathrm{Cu}(\mathrm{OTf})$.Tol, ethyl crotonate (4a) and 4g were purchased from Aldrich or Acros. $(R$ or $S)$-BINAP $($ L1), $(R$ or $S)$-Tol-BINAP (L2) and $(R)$-xylyl-BINAP $(\mathbf{L 3})$ were purchased from Stream. Unsaturated esters $(\boldsymbol{E})-\mathbf{2},(\boldsymbol{Z})-\mathbf{2}, \mathbf{4 c}$, $\mathbf{4 d},(E)-\mathbf{4 e},(Z)-\mathbf{4 e}$, and $\mathbf{4 g}$ were prepared from the corresponding aldehydes, via HornerEmmons reaction or Wittig-Horner reaction using well established protocols, and had identical properties (NMR) to those previously reported.

Grignard reagents were purchased from Aldrich (EtMgBr, $\mathrm{MeMgBr}$ ) or prepared from the corresponding alkyl bromides and magnesium turnings in $\mathrm{Et}_{2} \mathrm{O}$ following standard procedures. Grignard reagents were titrated using $s \mathrm{BuOH}$ and catalytic amounts of 1,10phenanthroline.

All reactions were conducted under nitrogen atmosphere using standard Schlenk techniques. Racemic 1,4-addition products were obtained by reaction of the enoates with the corresponding $\mathrm{RMgX}$ reagents $\left(-20{ }^{\circ} \mathrm{C}, \mathrm{Et}_{2} \mathrm{O}\right)$ and $\mathrm{CuI}(100 \mathrm{~mol} \%)$.

Analytical thin layer chromatography (TLC) was performed using Merck 60 F254 precoated silica gel plate $(0.2 \mathrm{~mm}$ thickness $)$. Subsequent to elution, plates were visualized using UV radiation $(254 \mathrm{~nm})$ on Spectroline Model ENF-24061/F $254 \mathrm{~nm}$. Further visualization was possible by staining with basic solution of potassium permanganate or

1. Apella, D. H.; Moritani, Y.; Shintani, R.; Ferreira, E. M.; Buchwald, S. L. J. Am. Chem. Soc. 1999, 121, 9473-9474. 
acidic solution of ceric molybdate.

Flash chromatography was performed using Merck silica gel 60 with freshly distilled solvents. Columns were typically packed as slurry and equilibrated with the appropriate solvent system prior to use.

Infrared spectra were recorded on a Bio-Rad FTS 165 FTIR spectrometer. The oil samples were examined under neat conditons.

High Resolution Mass (HRMS) spectra were obtained using Finnigan MAT95XP GC/HRMS (Thermo Electron Corporation).

Proton nuclear magnetic resonance spectra $\left({ }^{1} \mathrm{H}\right.$ NMR) were recorded on a Bruker Avance DPX 300 and Bruker AMX 400 spectrophotometer $\left(\mathrm{CDCl}_{3}\right.$ as solvent). Chemical shifts for ${ }^{1} \mathrm{H}$ NMR spectra are reported as $\delta$ in units of parts per million (ppm) downfield from $\mathrm{SiMe}_{4}(\delta 0.0)$ and relative to the signal of chloroform-d $(\delta 7.2600$, singlet). Multiplicities were given as: s (singlet); $\mathrm{d}$ (doublet); $\mathrm{t}$ (triplet); q (quartet); dd (doublets of doublet); ddd (doublets of doublets of doublet); dddd (doublets of doublets of doublets of doublet); dt (doublets of triplet); or $\mathrm{m}$ (multiplets). The number of protons (n) for a given resonance is indicated by $\mathrm{nH}$. Coupling constants are reported as a $J$ value in Hz. Carbon nuclear magnetic resonance spectra $\left({ }^{13} \mathrm{C}\right.$ NMR) are reported as $\delta$ in units of parts per million (ppm) downfield from $\mathrm{SiMe}_{4}(\delta 0.0)$ and relative to the signal of chloroform-d ( $\delta 77.03$, triplet). The proportion of diastereomers and geometric isomers was determined from the integration of ${ }^{1} \mathrm{H}$ NMR and ${ }^{13} \mathrm{C}$ NMR spectra.

Enantioselectivities were determined by capillary GC analysis (Chiraldex G-TA column (30 m x $0.25 \mathrm{~mm})$ ), using flame ionization detector or HPLC analysis employing a Daicel Chiracel ODH or OJ column at $25{ }^{\circ} \mathrm{C}$ (in comparison with racemic $(1,4)$-addition products). Optical rotations were measured in $\mathrm{CHCl}_{3}$ on a Schmidt + Haensdch polarimeter 
(Polartronic MH8) with a $10 \mathrm{~cm}$ cell (c given in $\mathrm{g} / 100 \mathrm{~mL}$ ). Absolute configuration of the products was determined by comparison with compounds previously published. 
Table I: Additions of EtMgBr to 2: Ligand effect ${ }^{a}$<smiles>COC(=O)/C=C/CCc1ccccc1</smiles>

2<smiles>Pc1ccc2ccccc2c1-c1c(P(P)P)ccc2ccccc12</smiles>

(R)-BINAP, L1<smiles>O=[P+]([O-])c1ccc2ccccc2c1-c1c([PH3+])ccc2ccccc12</smiles>

(R)-Tol-BINAP, L2<smiles>CC[C@H](CCc1ccccc1)CC(=O)OC</smiles>

$3 a$<smiles>Cc1ccc(C)cc1</smiles>

\begin{tabular}{|c|c|c|c|}
\hline entry & Catalyst & yield $(\%)^{b}$ & ee $(\%)^{c}$ \\
\hline 1 & $1 \mathrm{~mol} \% \mathrm{CuCl}+1.2 \mathrm{~mol} \% \mathbf{L} 2$ & 86 & 75 \\
\hline 2 & $1 \mathrm{~mol} \% \mathrm{CuBr}+1.2 \mathrm{~mol} \% \mathbf{L 2}$ & 83 & 70 \\
\hline 3 & $1 \mathrm{~mol} \% \mathrm{CuBr} \mathrm{SMe}_{2}+1.2 \mathrm{~mol} \% \mathbf{L} 2$ & 85 & 78 \\
\hline 4 & $1 \mathrm{~mol} \% \mathrm{CuI}+1.2 \mathrm{~mol} \% \mathbf{L 2}$ & 85 & 82 \\
\hline 5 & $1 \mathrm{~mol} \%(\mathrm{CuOTf}) \mathrm{Tol}_{0.5}+1.2 \mathrm{~mol} \% \mathbf{L 2}$ & 83 & 34 \\
\hline 6 & $1 \mathrm{~mol} \% \mathrm{CuI}+1.2 \mathrm{~mol} \% \mathbf{L 1}$ & 85 & 55 \\
\hline 7 & $0.5 \mathrm{~mol} \% \mathrm{1a}$ & 85 & 57 \\
\hline 8 & $1 \mathrm{~mol} \% \mathrm{CuI}+1.4 \mathrm{~mol} \% \mathbf{L} 2$ & 86 & 90 \\
\hline 9 & $1 \mathrm{~mol} \% \mathrm{CuI}+1.5 \mathrm{~mol} \% \mathbf{L} 2$ & 88 & 93 \\
\hline 10 & $1 \mathrm{~mol} \% \mathrm{CuI}+2.0 \mathrm{~mol} \% \mathbf{L} 2$ & 89 & 93 \\
\hline 11 & $0.5 \mathrm{~mol} \% \mathbf{1 a}+0.5 \mathrm{~mol} \% \mathbf{L} 2$ & 87 & 93 \\
\hline 12 & $1 \mathrm{~mol} \% \mathrm{CuI}+1.5 \mathrm{~mol} \% \mathbf{L 1}$ & 85 & 85 \\
\hline 13 & $1 \mathrm{~mol} \% \mathrm{CuI}+1.5 \mathrm{~mol} \% \mathbf{L} 3$ & 83 & 80 \\
\hline 14 & $0.5 \mathrm{~mol} \% 1 \mathrm{a}+1 \mathrm{~mol} \% \mathrm{Ph}_{3} \mathrm{P}$ & 85 & 53 \\
\hline 15 & $0.5 \mathrm{~mol} \% \mathbf{1 a}+1 \mathrm{~mol} \% \mathrm{Cy}_{3} \mathrm{P}$ & 88 & 83 \\
\hline 16 & $0.5 \mathrm{~mol} \% 1 \mathrm{a}+0.5 \mathrm{~mol} \%$ dppe & 86 & 65 \\
\hline
\end{tabular}

${ }^{a}$ All reactions were performed with $2(0.5 \mathrm{mmol}), \mathrm{EtMgBr}\left(2.0 \mathrm{mmol}, 3 \mathrm{M}\right.$ in ether) in $t$-BuOMe $(1 \mathrm{~mL})$ at $-40^{\circ} \mathrm{C}$ unless otherwise stated. ${ }^{b}$ Isolated yield. ${ }^{c}$ Determined by HPLC analysis employing a Daicel Chiracel ODH column 
Figure 1 The relationship of ee with the ratio of $(R)$-Tol-BINAP : CuI

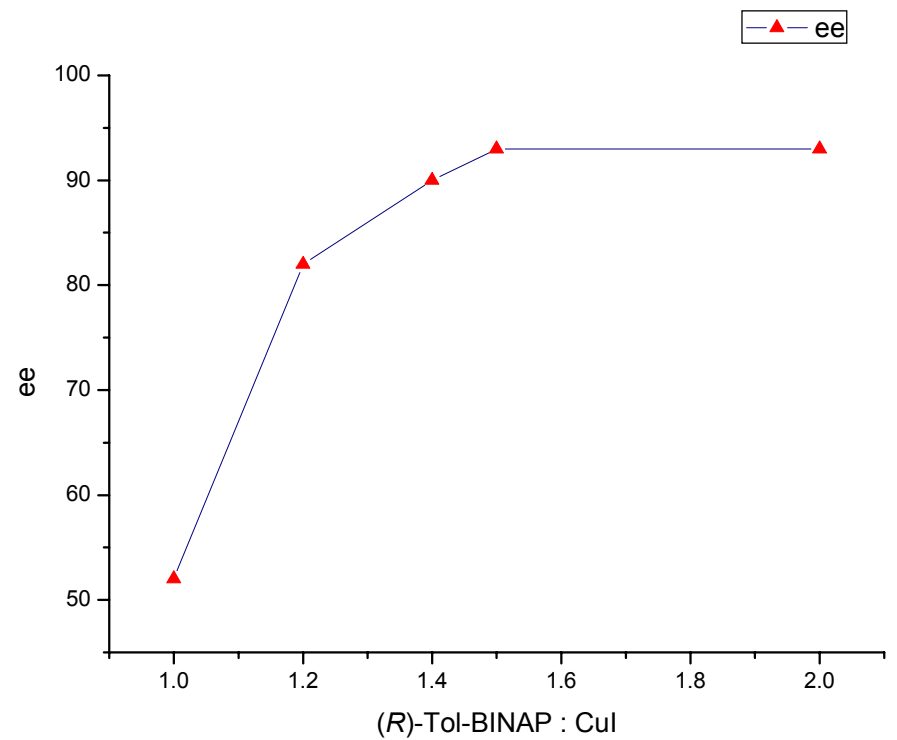

${ }^{a}$ All reactions were performed with $2(0.5 \mathrm{mmol}), \mathrm{EtMgBr}(2.0 \mathrm{mmol}, 3 \mathrm{M}$ in ether $)$ in $t$-BuOMe $(1 \mathrm{~mL})$ at $-40{ }^{\circ} \mathrm{C}$. 


\section{Preparation and characterization of $\mathrm{Cu}$-complexes 1a.}

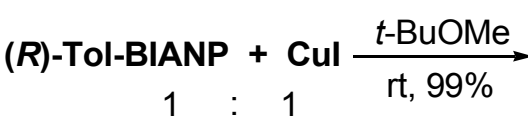<smiles>O=[N+]([O-])c1ccc2ccccc2c1-c1c(Br)ccc2ccccc12</smiles><smiles></smiles>

1a
$(R)$-Tol-BIANP $=C_{P}$

A solution of $(\boldsymbol{R})$-Tol-BINAP $(67.9 \mathrm{mg}, 0.1 \mathrm{mmol})$ and $\mathrm{CuI}(19.0 \mathrm{mg}, 0.1 \mathrm{mmol})$ in $t$-BuOMe $(10 \mathrm{~mL})$ in a Schlenk tube was stirred at room temperature (RT) for $1 \mathrm{~h}$. The solvent was removed under vacuum and the resulting orange crude residue was washed with cold pentane $(3 \times 2 \mathrm{~mL})$ to afford $\mathbf{1 a}$ as an light orange powder (86.0 mg, 99\% yield, $\mathrm{R}_{\mathrm{f}}=0.35$ (25\% Ethyl acetate / Hexane)].

FTIR (KBr, neat): $v$ 3032, 2960, 2918, 2860, 1597, 1498, 1396, 1261, 1093, 1020, 806, 744;

${ }^{1} \mathbf{H}$ NMR (300 MHz, CDCl$\left.)_{3}\right): \delta 8.00$ (m, 8H), 7.43-7.48 (m, 8H), 7.26-7.30 (m, 10H), 7.22-7.23 (m, 8H), 7.267.01-7.06 (m, 12H), $6.82(\mathrm{~d}, J=8.4 \mathrm{~Hz}, 4 \mathrm{H}), 6.23(\mathrm{~d}, J=7.5 \mathrm{~Hz}, 4 \mathrm{H}), 2.29$ (s, 12H), 1.90 (s, 12H);

${ }^{13} \mathbf{C}$ NMR (75.4 MHz, $\left.\mathbf{C D C l}_{3}\right): \delta$ 140.8, 139.0, 138.7, 135.6, 133.3, 133.1, 132.3, 129.8, 128.2, 128.0, 127.8, $127.4,126.3,126.0,21.4,21.0$.

${ }^{31} \mathbf{P}$ NMR ( $\left.\mathbf{M H z}, \mathbf{C D C l}_{3}\right): \delta \quad-6.5$.

HRMS calcd. For $\mathrm{C}_{96} \mathrm{H}_{81} \mathrm{Cu}_{2} \mathrm{I}_{1} \mathrm{P}_{4}\left[\mathrm{M}^{+}-\mathrm{I}+\mathrm{H}\right] 1610.2925$, found 1610.2877; 


\section{General Procedure for the Enantioselective Conjugate Addition}

In a Schlenk tube equipped with septum and stirring bar, $(R)$-Tol-BINAP (5.1 mg, $0.0075 \mathrm{mmol})$ and $\mathrm{CuI}$ $(0.95 \mathrm{mg}, 0.005 \mathrm{mmol})$ was dissolved in $t$-BuOMe $(1.0 \mathrm{~mL})$ and stirred under nitrogen at room temperature until yellow suspension was observed. The mixture was then cooled to $-40{ }^{\circ} \mathrm{C}$ and EtMgBr (Aldrich, $3.0 \mathrm{M}$ solution in $\left.\mathrm{Et}_{2} \mathrm{O}, 2.5 \mathrm{mmol}\right)$ was added. After stirring for $5 \mathrm{~min}$, a solution of unsaturated ester $(0.5 \mathrm{mmol}) \mathrm{in} t$-BuOMe $(0.25 \mathrm{~mL})$ was added dropwise over $1 \mathrm{~h}$ by syringe pump. After stirring at $-40{ }^{\circ} \mathrm{C}$ for 2 to 3 hours, $\mathrm{MeOH}(0.5$ $\mathrm{mL})$ and $\mathrm{NH}_{4} \mathrm{Cl}(1 \mathrm{M}, 2 \mathrm{~mL})$ were sequentially added, and the mixture was warmed to room temperature (RT). After extracted with $\mathrm{Et}_{2} \mathrm{O}(5 \mathrm{~mL} \times 3)$, the combined organic phases were dried and concentrated to a yellow oil which was purified by flash chromatograph $\left(1: 99 \mathrm{Et}_{2} \mathrm{O}\right.$ /pentane) to yield desired product.

Racemic 1,4-addition products were obtained by the reaction of the enoates with the corresponding Grignard reagents $\left(-20{ }^{\circ} \mathrm{C}, \mathrm{Et}_{2} \mathrm{O}\right)$ and $\mathrm{CuI}(100 \mathrm{~mol} \%)$.

\section{NOTES:}

- $\mathrm{GC}$ analysis was carried out on a sample obtained after aqueous extraction with $\mathrm{Et}_{2} \mathrm{O}$, which has been passed through a short plug of silica gel. 


\section{Characterization of CA products:}

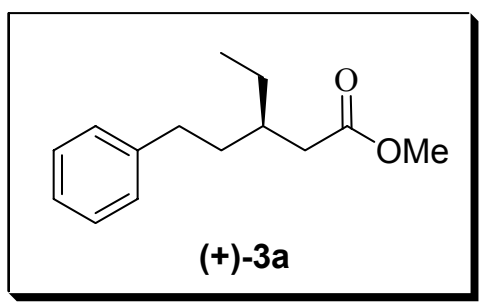

(+)-Methyl 3-phenethylpentanoate, $\mathbf{R}_{f}=0.72(\mathrm{EA} / \mathrm{Hexane}=1 / 4)$;

(Table 3, entry 6): colorless oil, $86 \%$ yield, $94 \%$ ee, $[\alpha]_{\mathrm{D}}{ }^{22}=+7.7(c=2.5)$, lit. $^{2}:[\alpha]_{\mathrm{D}}{ }^{20}=+7.7$;

FTIR (KBr, neat): $v 1737(\mathrm{C}=\mathrm{O})$;

${ }^{1}$ H NMR (300 MHz, CDCl $): \delta$ 7.16-7.30 (m, 5H), $3.66(\mathrm{~s}, 3 \mathrm{H}), 2.60(\mathrm{t}, J=8.2 \mathrm{~Hz}, 2 \mathrm{H}), 2.31(\mathrm{~d}, J=6.8 \mathrm{~Hz}$, 2H), 1.84-1.93 (m, 1H), 1.57-1.66 (m, 2H), 1.38-1.47 (m, 2H), 0.90 (t, J=7.4 Hz, 3H);

${ }^{13}$ C NMR (75.4 MHz, $\left.\mathbf{C D C l}_{3}\right): \delta 173.8(\mathrm{C}), 142.5(\mathrm{C}), 128.3(\mathrm{CH}), 125.7(\mathrm{CH}), 51.4\left(\mathrm{CH}_{3}\right), 38.5\left(\mathrm{CH}_{2}\right), 36.3$ $(\mathrm{CH}), 35.4\left(\mathrm{CH}_{2}\right), 33.0\left(\mathrm{CH}_{2}\right), 26.2\left(\mathrm{CH}_{2}\right), 10.7\left(\mathrm{CH}_{3}\right)$;

HRMS calcd. For $\mathrm{C}_{14} \mathrm{H}_{20} \mathrm{O}_{2} 220.1463$, found 220.1457;

The enantiomeric excess was determined by HPLC analysis employing a Daicel Chiracel ODH column (Hexane : $i$-propanol $99.2: 0.8,1 \mathrm{~mL} / \mathrm{min}$ ): $\mathrm{t}_{1}=6.22 \mathrm{~min}$ (minor), $\mathrm{t}_{2}=7.29 \mathrm{~min}$ (major).

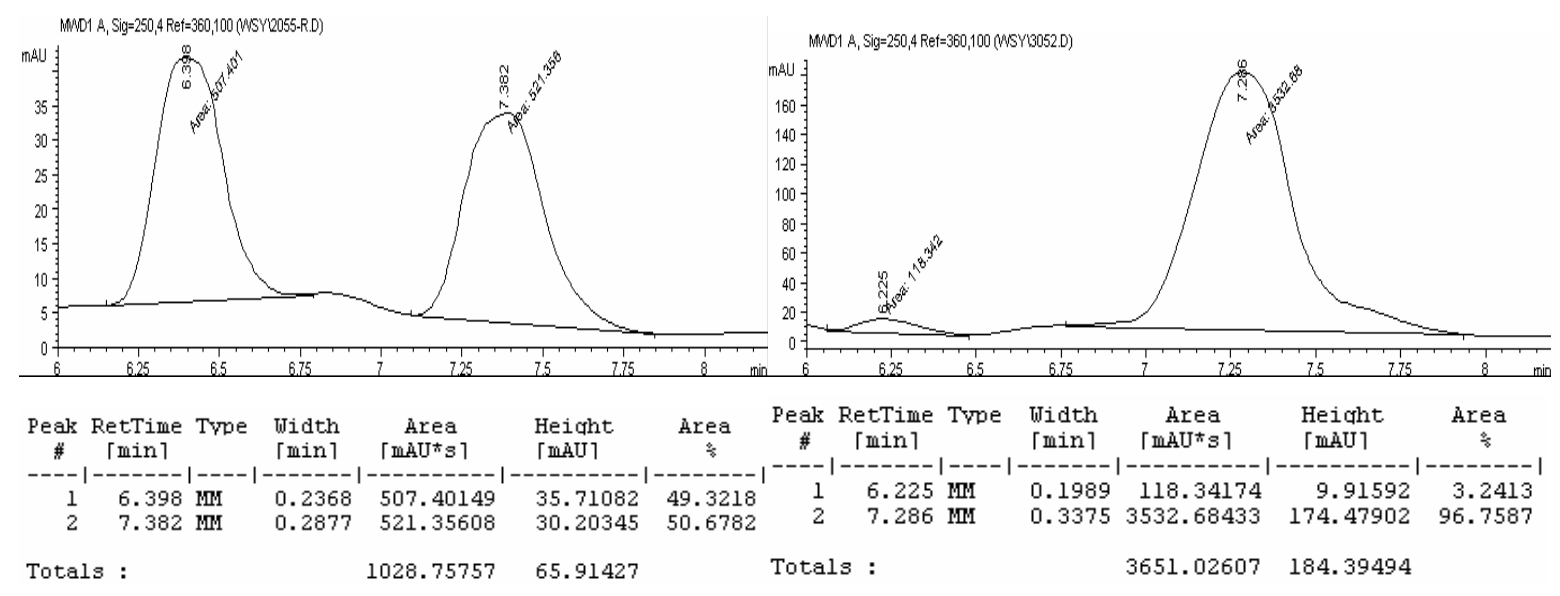

2. López, F.; Harutyunyan, S. R.; Minnaard, A. J.; Feringa, B. L. Angew. Chem., Int. Ed. 2005, $44,2752-2756$. 


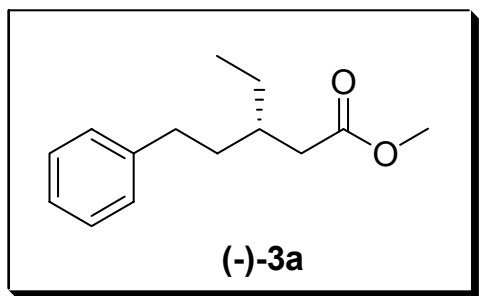

(-)-Methyl 3-phenethylpentanoate, $\mathbf{R}_{f}=0.72(\mathrm{EA} /$ Hexane $=1 / 4)$;

(Figure 1): colorless oil, $86 \%$ yield, $94 \%$ ee, $[\alpha]_{\mathrm{D}}^{22}=-5.4(c=1.0)$;

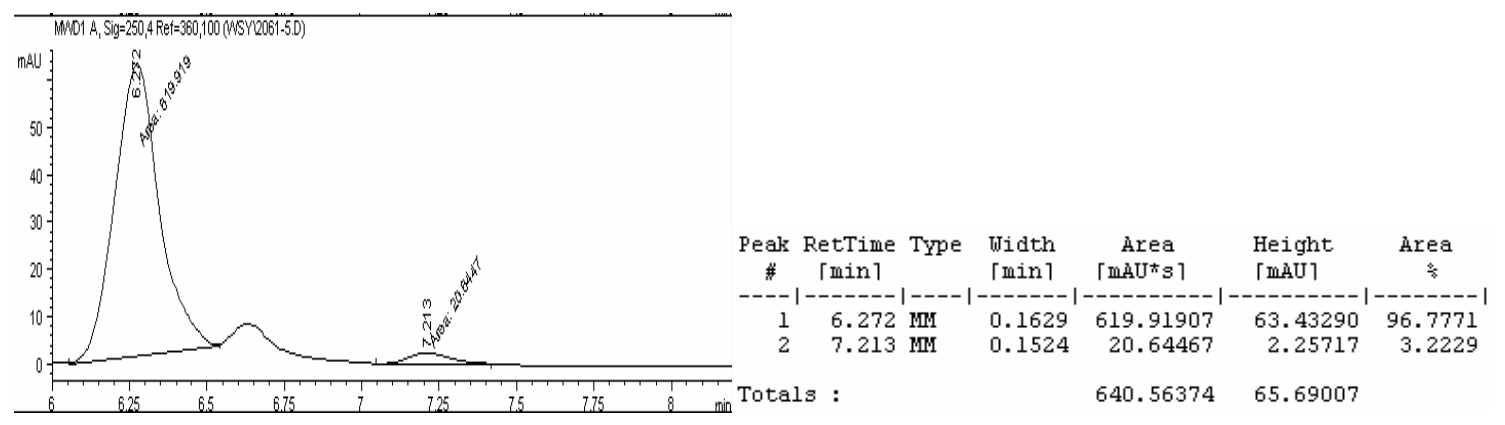

The enantiomeric excess was determined by HPLC analysis employing a Daicel Chiracel ODH column (Hexane : $i$-propanol $99.2: 0.8,1 \mathrm{~mL} / \mathrm{min}$ ): $\mathrm{t}_{1}=6.27$ min (major), $\mathrm{t}_{2}=7.21 \mathrm{~min}$ (minor). 


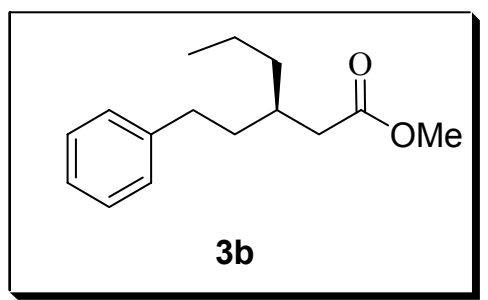

(+)-Methyl 3-phenethylhexanoate, $\mathbf{R}_{\boldsymbol{f}}=0.70(\mathrm{EA} / \mathrm{Hexane}=1 / 4)$;

(Table 2, entry 2): colorless oil, $90 \%$ yield, $92 \%$ ee, $[\alpha]_{\mathrm{D}}{ }^{22}=+5.1(c=3.7)$;

FTIR (KBr, neat): $v 1735(\mathrm{C}=\mathrm{O})$;

${ }^{1}$ H NMR (300 MHz, CDCl $): \delta$ 7.15-7.29 (m, 5H), $3.65(\mathrm{~s}, 3 \mathrm{H}), 2.60$ (t, J=8.2 Hz, 2H), $2.31(\mathrm{~d}, J=6.8 \mathrm{~Hz}$, $2 \mathrm{H}), 1.92-1.96(\mathrm{~m}, 1 \mathrm{H}), 1.55-1.66(\mathrm{~m}, 2 \mathrm{H}), 1.29-1.38(\mathrm{~m}, 4 \mathrm{H}), 0.89(\mathrm{t}, J=6.9 \mathrm{~Hz}, 3 \mathrm{H})$;

${ }^{13}$ C NMR (75.4 MHz, $\left.\mathbf{C D C l}_{3}\right): \delta 173.8(\mathrm{C}), 142.5(\mathrm{C}), 128.5(\mathrm{CH}), 125.7(\mathrm{CH}), 51.4\left(\mathrm{CH}_{3}\right), 38.9\left(\mathrm{CH}_{2}\right), 36.1$ $\left(\mathrm{CH}_{2}\right), 35.9\left(\mathrm{CH}_{2}\right), 34.7(\mathrm{CH}), 33.0\left(\mathrm{CH}_{2}\right), 19.6\left(\mathrm{CH}_{2}\right), 14.3\left(\mathrm{CH}_{3}\right)$;

HRMS calcd. For $\mathrm{C}_{15} \mathrm{H}_{22} \mathrm{O}_{2} 234.1620$, found 234.1607;

The enantiomeric excess was determined by HPLC analysis employing a Daicel Chiracel ODH column (Hexane : $i$-propanol $99.2: 0.8,1 \mathrm{~mL} / \mathrm{min}$ ): $\mathrm{t}_{1}=5.85 \mathrm{~min}$ (minor), $\mathrm{t}_{2}=6.66 \mathrm{~min}$ (major).

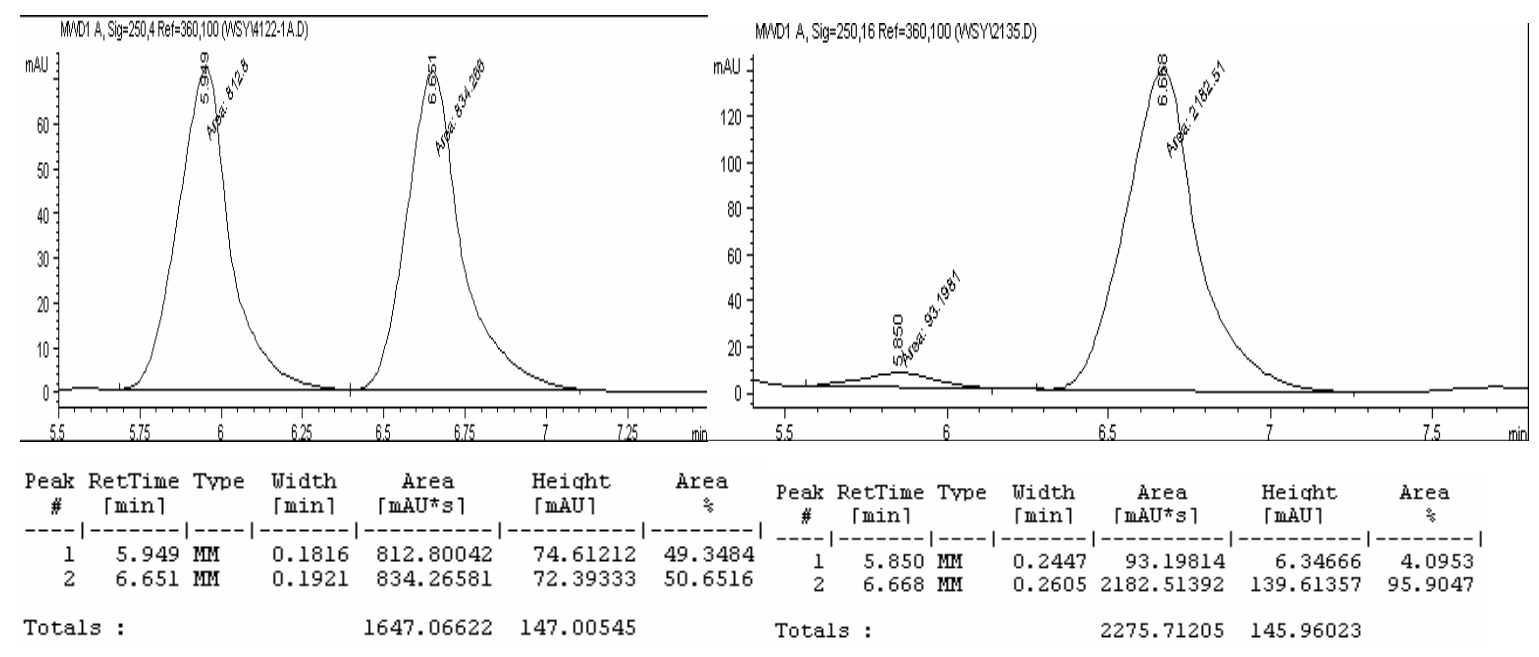




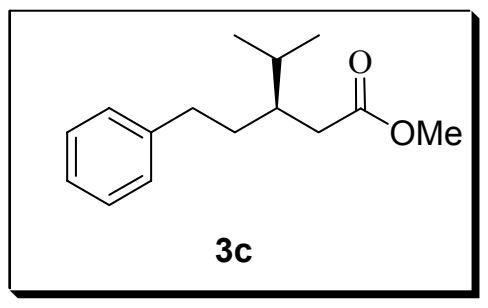

(+)-Methyl 4-methyl-3-phenethylpentanoate, $\mathbf{R}_{\boldsymbol{f}}=0.71(\mathrm{EA} / \mathrm{Hexane}=1 / 4)$;

(Table 2, entry 3): colorless oil, $89 \%$ yield, $91 \%$ ee, $[\alpha]_{\mathrm{D}}^{23}=+6.2(c=1.0)$;

FTIR (KBr, neat): $v 1737(\mathrm{C}=\mathrm{O})$;

${ }^{1}$ H NMR (300 MHz, $\left.\mathbf{C D C l}_{3}\right): \delta 7.16-7.30(\mathrm{~m}, 5 \mathrm{H}), 3.66(\mathrm{~s}, 3 \mathrm{H}), 2.59(\mathrm{t}, J=8.2 \mathrm{~Hz}, 2 \mathrm{H}), 2.35(\mathrm{dd}, J=15.0$ and $6.1 \mathrm{~Hz}, 1 \mathrm{H}), 2.23(\mathrm{dd}, J=15.0$ and $7.3 \mathrm{~Hz}, 1 \mathrm{H}), 1.65-1.88(\mathrm{~m}, 2 \mathrm{H}), 1.48-1.63(\mathrm{~m}, 2 \mathrm{H}), 0.87(\mathrm{t}, J=6.9 \mathrm{~Hz}, 6 \mathrm{H})$; ${ }^{13}$ C NMR (75.4 MHz, $\left.\mathbf{C D C l}_{3}\right): \delta 174.3(\mathrm{C}), 142.6(\mathrm{C}), 128.3(\mathrm{CH}), 125.7(\mathrm{CH}), 51.5\left(\mathrm{CH}_{3}\right), 40.7(\mathrm{CH}), 35.9$ $\left(\mathrm{CH}_{2}\right), 33.7\left(\mathrm{CH}_{2}\right), 33.3\left(\mathrm{CH}_{2}\right), 29.9(\mathrm{CH}), 19.3\left(\mathrm{CH}_{3}\right), 18.5\left(\mathrm{CH}_{3}\right)$;

HRMS calcd. For $\mathrm{C}_{15} \mathrm{H}_{22} \mathrm{O}_{2} 234.1620$, found 234.1606;

The enantiomeric excess was determined by HPLC analysis employing a Daicel Chiracel ODH column (Hexane : $i$-propanol $99.2: 0.8,1 \mathrm{~mL} / \mathrm{min}$ ): $\mathrm{t}_{1}=5.61 \mathrm{~min}$ (minor), $\mathrm{t}_{2}=6.48 \mathrm{~min}$ (major).
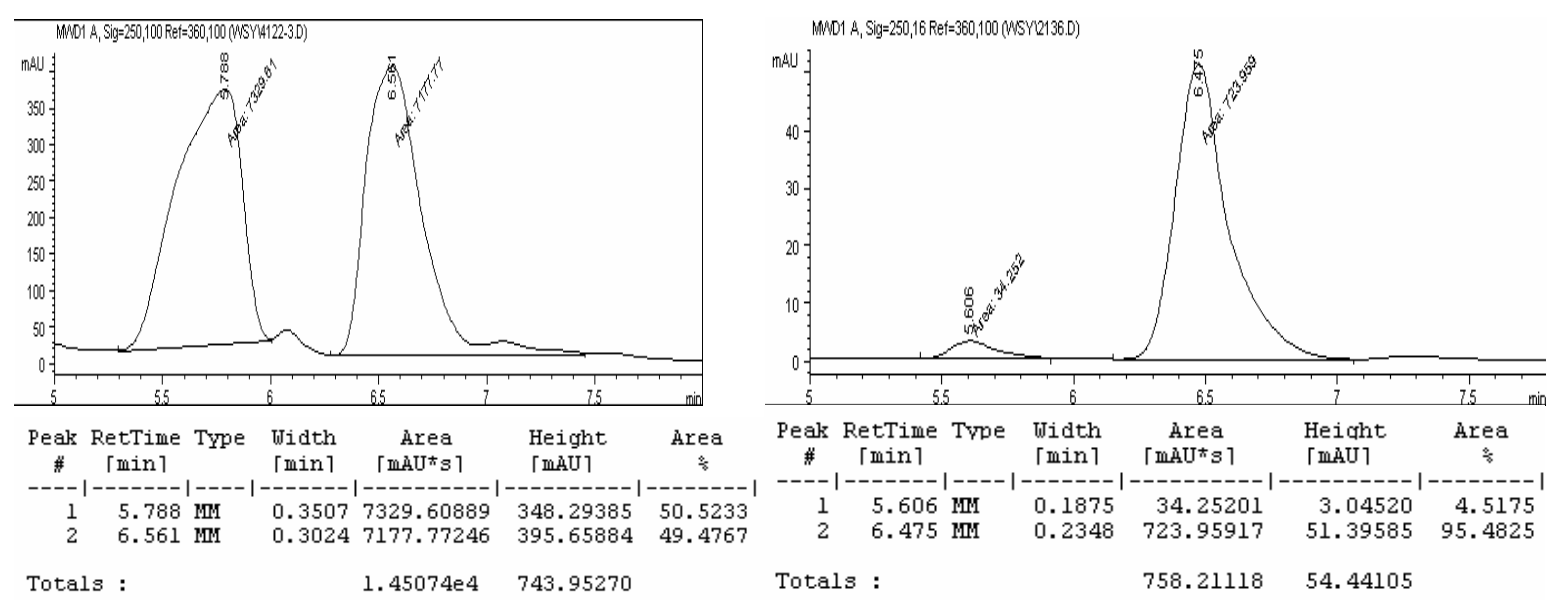


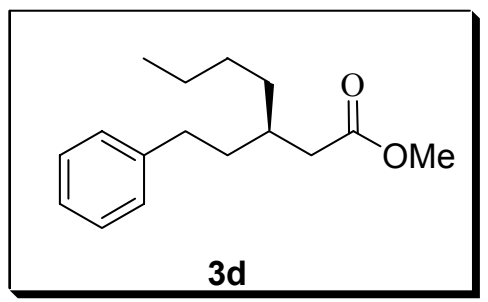

(+)-Methyl 3-phenethylheptanoate $\mathbf{R}_{f}=0.71($ EA/Hexane $=1 / 4)$

(Table 2, entry 4$)$, colorless oil, $90 \%$ yield, $92 \%$ ee, $[\alpha]_{\mathrm{D}}{ }^{24}=+6.2(c=2.2)$;

FTIR (KBr, neat): $v 1735(\mathrm{C}=\mathrm{O})$;

${ }^{1} \mathbf{H}$ NMR (300 MHz, CDCl$\left.)_{3}\right): \delta$ 7.18-7.32 (m, 5H), $3.68(\mathrm{~s}, 3 \mathrm{H}), 2.62(\mathrm{t}, J=8.2 \mathrm{~Hz}, 2 \mathrm{H}), 2.33(\mathrm{~d}, J=6.8 \mathrm{~Hz}$, $2 \mathrm{H}), 1.91-1.97(\mathrm{~m}, 1 \mathrm{H}), 1.54-1.69(\mathrm{~m}, 2 \mathrm{H}), 1.31-1.41(\mathrm{~m}, 4 \mathrm{H}), 0.95(\mathrm{t}, J=7.2 \mathrm{~Hz}, 3 \mathrm{H})$;

${ }^{13}$ C NMR (75.4 MHz, $\left.\mathbf{C D C l}_{3}\right): \delta 173.9(\mathrm{C}), 142.5(\mathrm{C}), 128.3(\mathrm{CH}), 125.7(\mathrm{CH}), 51.4\left(\mathrm{CH}_{3}\right), 38.9\left(\mathrm{CH}_{2}\right), 35.8$ $\left(\mathrm{CH}_{2}\right), 34.8(\mathrm{CH}), 33.4\left(\mathrm{CH}_{2}\right), 33.0\left(\mathrm{CH}_{2}\right), 28.7\left(\mathrm{CH}_{2}\right), 22.9\left(\mathrm{CH}_{2}\right), 14.0\left(\mathrm{CH}_{3}\right)$;

HRMS calcd. For $\mathrm{C}_{16} \mathrm{H}_{24} \mathrm{O}_{2} 248.1776$, found 248.1767;

The enantiomeric excess was determined by HPLC analysis employing a Daicel Chiracel ODH column (Hexane : $i$-propanol $99.2: 0.8,1 \mathrm{~mL} / \mathrm{min}$ ): $\mathrm{t}_{1}=5.55 \mathrm{~min}$ (minor), $\mathrm{t}_{2}=6.29 \mathrm{~min}$ (major).

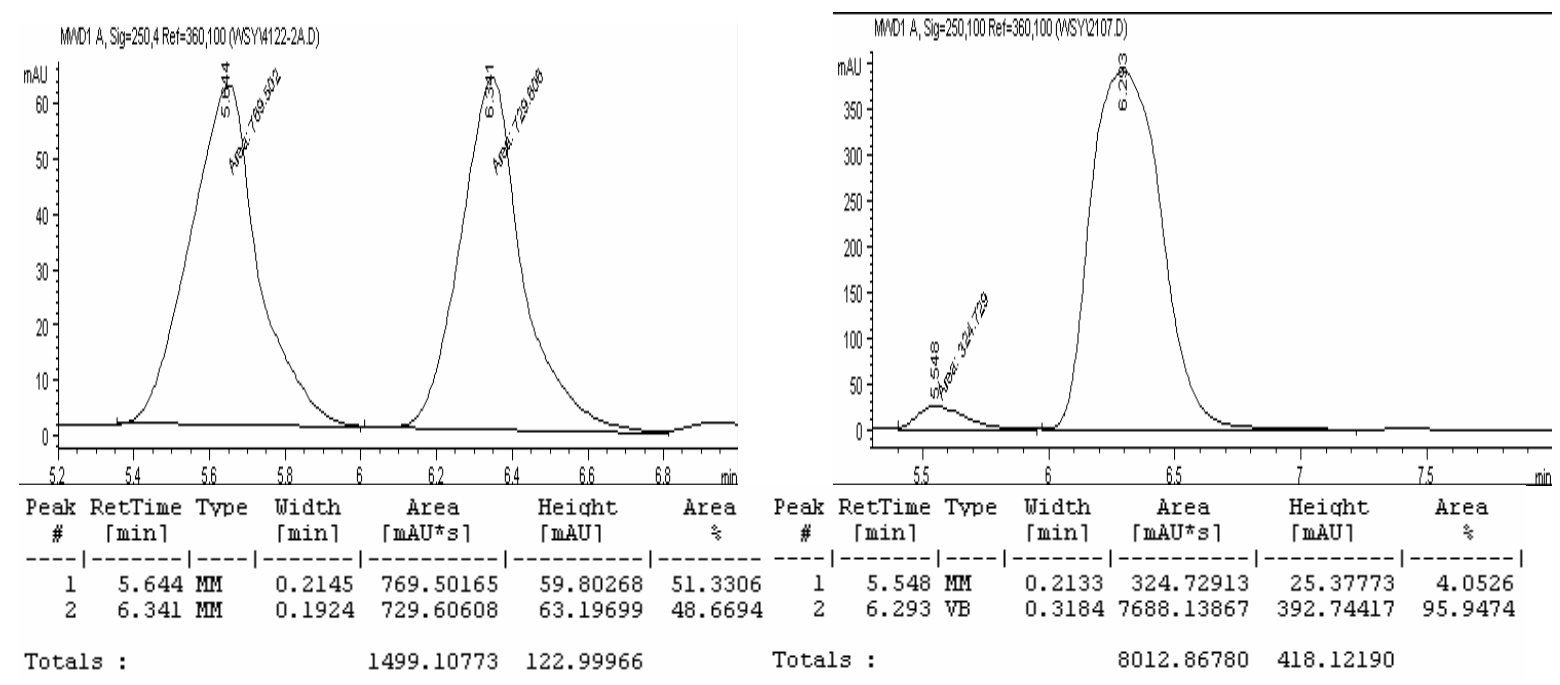




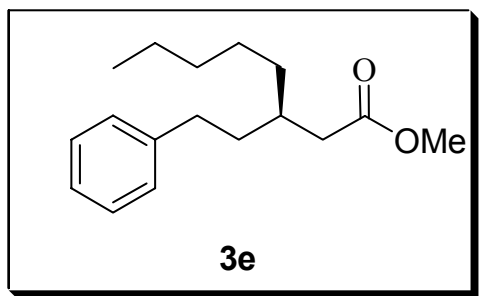

$(+)$-Methyl 3-phenethylpentanoate, $\mathbf{R}_{\boldsymbol{f}}=0.72(\mathrm{EA} /$ Hexane $=1 / 4)$;

(Table 2, entry 5): colorless oil, $86 \%$ yield, $90 \%$ ee, $[\alpha]_{\mathrm{D}}{ }^{20}=+4.6(c=1.0)$;

FTIR (KBr, neat): $v 1737(\mathrm{C}=\mathrm{O})$;

${ }^{1}$ H NMR (300 MHz, CDCl $): \delta$ 7.16-7.28 (m, 5H), $3.66(\mathrm{~s}, 3 \mathrm{H}), 2.61(\mathrm{t}, J=8.2 \mathrm{~Hz}, 2 \mathrm{H}), 2.29(\mathrm{~d}, J=6.8 \mathrm{~Hz}$, 2H), $1.86(\mathrm{~m}, 1 \mathrm{H}), 1.61(\mathrm{~m}, 2 \mathrm{H}), 1.39(\mathrm{~m}, 2 \mathrm{H}), 0.88(\mathrm{t}, J=6.8 \mathrm{~Hz}, 3 \mathrm{H})$;

${ }^{13}$ C NMR (75.4 MHz, $\left.\mathbf{C D C l}_{3}\right): \delta 173.8(\mathrm{C}), 142.5(\mathrm{C}), 128.3(\mathrm{CH}), 125.7(\mathrm{CH}), 51.4\left(\mathrm{CH}_{3}\right), 38.9\left(\mathrm{CH}_{2}\right), 35.8$ $\left(\mathrm{CH}_{2}\right), 34.9(\mathrm{CH}), 33.7\left(\mathrm{CH}_{2}\right), 33.0\left(\mathrm{CH}_{2}\right), 32.0\left(\mathrm{CH}_{2}\right), 26.1\left(\mathrm{CH}_{2}\right), 22.6\left(\mathrm{CH}_{2}\right), 14.0\left(\mathrm{CH}_{3}\right)$;

HRMS calcd. For $\mathrm{C}_{17} \mathrm{H}_{26} \mathrm{O}_{2} 262.1933$, found 262.1961;

The enantiomeric excess was determined by HPLC analysis employing a Daicel Chiracel ODH column (Hexane : $i$-propanol $99.2: 0.8,1 \mathrm{~mL} / \mathrm{min}$ ): $\mathrm{t}_{1}=5.42 \mathrm{~min}$ (minor), $\mathrm{t}_{2}=6.34 \mathrm{~min}$ (major).

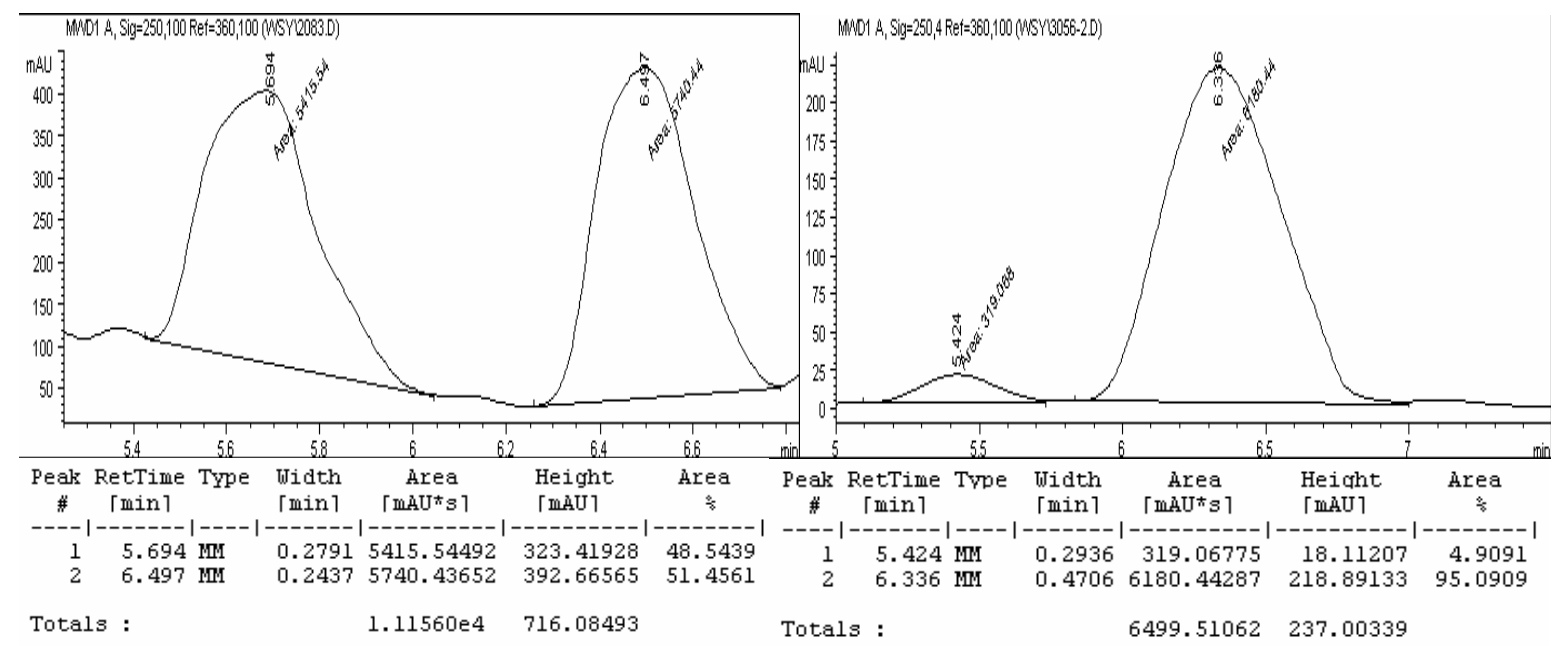




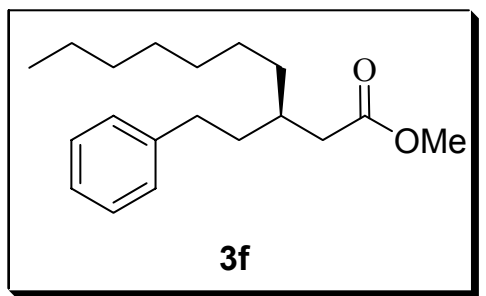

$(+)$-Methyl 3-phenethylpentanoate, $\mathbf{R}_{\boldsymbol{f}}=0.71(\mathrm{EA} /$ Hexane $=1 / 4)$;

(Table 2, entry 6): colorless oil, $89 \%$ yield, $92 \%$ ee, $[\alpha]_{\mathrm{D}}{ }^{24}=+3.7(c=2.2)$;

FTIR (KBr, neat): $v 1737(\mathrm{C}=\mathrm{O})$;

${ }^{1}$ H NMR (300 MHz, CDCl 3 ): $\delta$ 7.18-7.32 (m, 5H), $3.68(\mathrm{~s}, 3 \mathrm{H}), 2.63(\mathrm{t}, J=8.2 \mathrm{~Hz}, 2 \mathrm{H}), 2.33(\mathrm{~d}, J=6.8 \mathrm{~Hz}$, 2H), 1.93-1.95 (m, 1H), 1.60-1.69 (m, 2H), $1.29(\mathrm{~m}, 12 \mathrm{H}), 0.91(\mathrm{t}, J=6.9 \mathrm{~Hz}, 3 \mathrm{H})$;

${ }^{13} \mathbf{C}$ NMR (75.4 MHz, $\left.\mathbf{C D C l}_{3}\right): \delta 173.8(\mathrm{C}), 142.5(\mathrm{C}), 128.3(\mathrm{CH}), 125.7(\mathrm{CH}), 51.4\left(\mathrm{CH}_{3}\right), 38.9\left(\mathrm{CH}_{2}\right), 35.8$ $\left(\mathrm{CH}_{2}\right), 34.9(\mathrm{CH}), 33.8\left(\mathrm{CH}_{2}\right), 33.0\left(\mathrm{CH}_{2}\right), 31.9\left(\mathrm{CH}_{2}\right), 29.8\left(\mathrm{CH}_{2}\right), 29.3\left(\mathrm{CH}_{2}\right), 26.5\left(\mathrm{CH}_{2}\right), 22.7\left(\mathrm{CH}_{2}\right), 14.1$ $\left(\mathrm{CH}_{3}\right)$;

HRMS calcd. For $\mathrm{C}_{19} \mathrm{H}_{30} \mathrm{O}_{2} 290.2246$, found 290.2240;

The enantiomeric excess was determined by HPLC analysis employing a Daicel Chiracel ODH column (Hexane : $i$-propanol $99.2: 0.8,1 \mathrm{~mL} / \mathrm{min}$ ): $\mathrm{t}_{1}=5.39 \mathrm{~min}$ (minor), $\mathrm{t}_{2}=6.12$ (major).
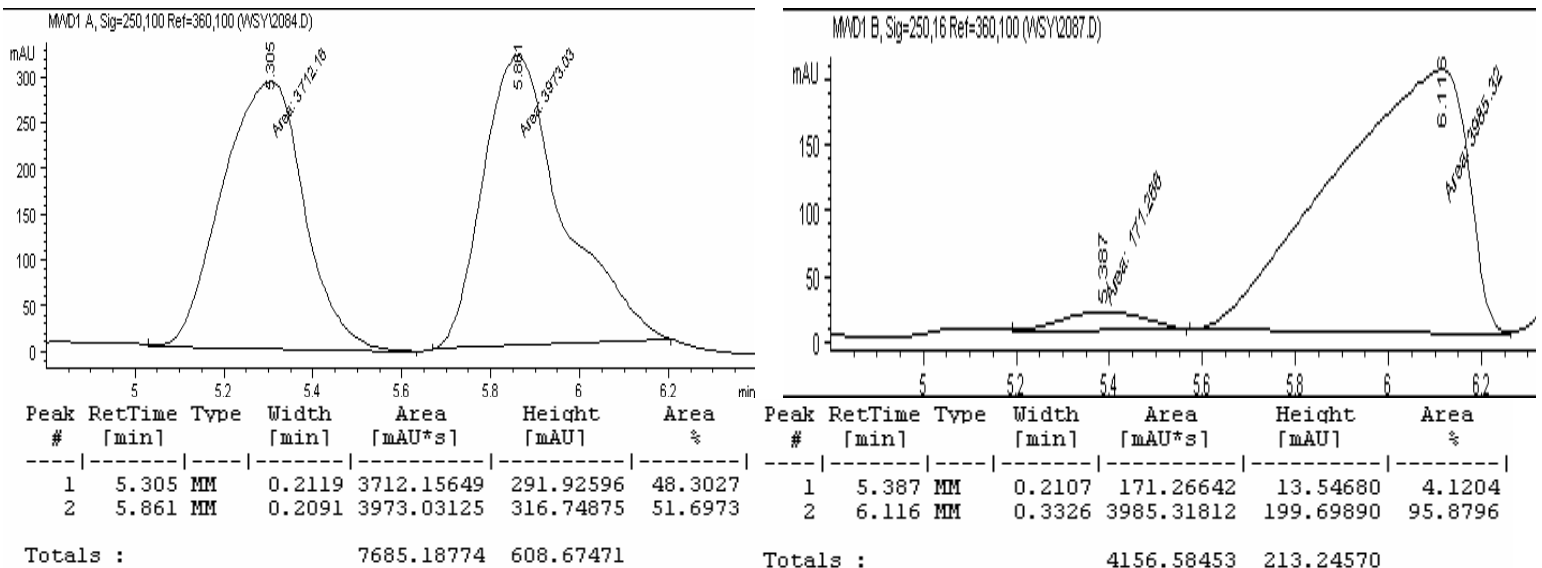


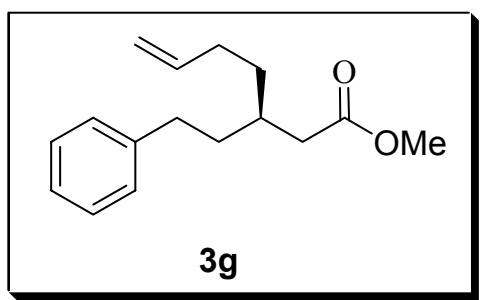

(+)-Methyl 3-phenethylhept-6-enoate

(Table 1, entry 7): colorless oil, $90 \%$ yield, $94 \%$ ee, $[\alpha]_{\mathrm{D}}{ }^{20}=+7.9(c=0.9)$;

FTIR (KBr, neat): $v 1737(\mathrm{C}=\mathrm{O})$;

${ }^{1}$ H NMR (300 MHz, $\left.\mathbf{C D C l}_{3}\right): \delta$ 7.14-7.29 (m, 5H), 5.74-5.83 (m, 1H), 4.92-5.03 (m, 2H), 3.65 (s, 3H), 2.60 (t, J $=8.2 \mathrm{~Hz}, 2 \mathrm{H}), 2.34(\mathrm{~d}, J=6.8 \mathrm{~Hz}, 2 \mathrm{H}), 2.06(\mathrm{dd}, J=14.4$ and $7.8 \mathrm{~Hz}, 1 \mathrm{H}), 1.91-2.00(\mathrm{~m}, 1 \mathrm{H}), 1.60-1.67(\mathrm{~m}$, $2 \mathrm{H}), 1.40-1.50(\mathrm{dt}, J=14.8$ and $7.1 \mathrm{~Hz}, 2 \mathrm{H})$;

${ }^{13}$ C NMR (75.4 MHz, $\left.\mathbf{C D C l}_{3}\right): \delta 173.6(\mathrm{C}), 142.4(\mathrm{C}), 138.5(\mathrm{CH}), 128.3(\mathrm{CH}), 128.3(\mathrm{CH}), 125.8(\mathrm{CH})$, 114.6( $\left(\mathrm{CH}_{2}\right), 51.5\left(\mathrm{CH}_{3}\right), 38.7\left(\mathrm{CH}_{2}\right), 35.7\left(\mathrm{CH}_{2}\right), 34.4(\mathrm{CH}), 33.0\left(\mathrm{CH}_{2}\right), 33.0\left(\mathrm{CH}_{2}\right), 30.8\left(\mathrm{CH}_{2}\right)$;

HRMS calcd. For $\mathrm{C}_{16} \mathrm{H}_{22} \mathrm{O}_{2} 246.1620$, found 246.1615;

The enantiomeric excess was determined by chiral HPLC analysis, Chiralcel OJ (Hexane : $i$-propanol $99.7: 0.3$, $1 \mathrm{~mL} / \mathrm{min}$ ): $\mathrm{t}_{1}=36.88 \min$ (minor), $\mathrm{t}_{2}=39.02$ (major).

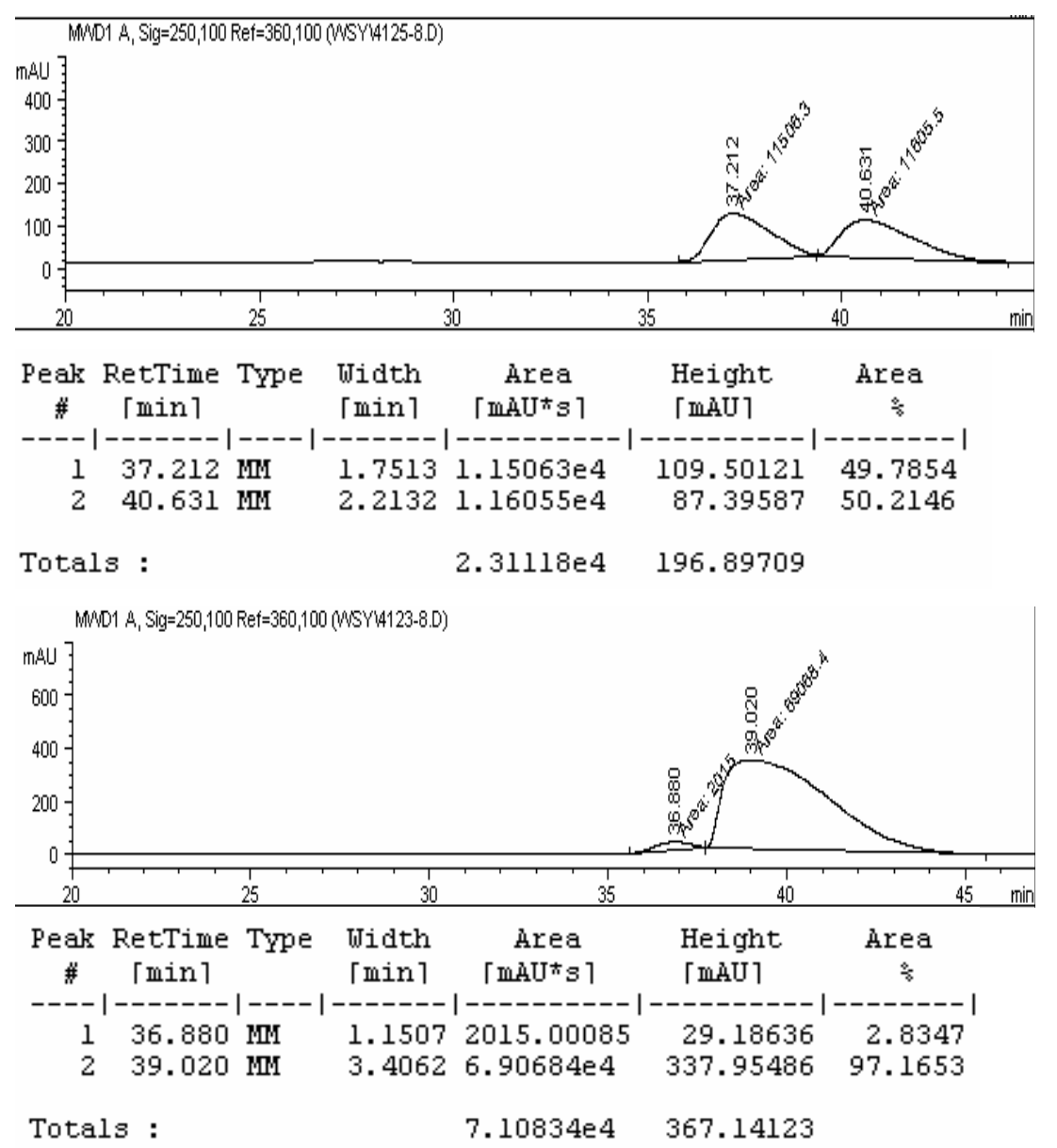




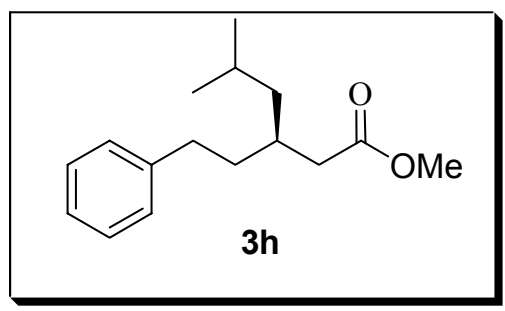

(-)-Methyl 5-methyl-3-phenethylhexanoate

(Table 2, entry 8): colorless oil, $91 \%$ yield, $86 \%$ ee, $[\alpha]_{\mathrm{D}}^{20}=+7.0(c=1.0)$;

FTIR (KBr, neat): $v 1737(\mathrm{C}=\mathrm{O})$;

${ }^{1}$ H NMR (300 MHz, CDCl 3 ): $\delta$ 7.16-7.30 (m, 5H), $3.65(\mathrm{~s}, 3 \mathrm{H}), 2.60(\mathrm{t}, J=8.2 \mathrm{~Hz}, 1 \mathrm{H}), 2.31(\mathrm{~d}, J=6.6 \mathrm{~Hz}$, 2H), 1.95-2.04 (m, 1H), 1.57-1.68 (m, 3H), 1.12-1.30 (m, 2H), $0.88(\mathrm{~d}, J=6.6 \mathrm{~Hz}, 6 \mathrm{H})$;

${ }^{13}$ C NMR (75.4 MHz, $\left.\mathbf{C D C l}_{3}\right): \delta 173.8(\mathrm{C}), 142.5(\mathrm{C}), 128.3(\mathrm{CH}), 125.7(\mathrm{CH}), 51.4\left(\mathrm{CH}_{3}\right), 43.6\left(\mathrm{CH}_{2}\right), 39.1$ $\left(\mathrm{CH}_{2}\right), 36.1\left(\mathrm{CH}_{2}\right), 32.8\left(\mathrm{CH}_{2}\right), 32.7(\mathrm{CH}), 25.3(\mathrm{CH}), 22.8\left(\mathrm{CH}_{3}\right), 22.7\left(\mathrm{CH}_{3}\right)$;

HRMS calcd. For $\mathrm{C}_{16} \mathrm{H}_{24} \mathrm{O}_{2} 248.1776$, found 248.1760;

The enantiomeric excess was determined by chiral HPLC analysis, Chiralcel OJ (Hexane : $i$-propanol $99.5: 0.5$, $1 \mathrm{~mL} / \mathrm{min}$ ): $\mathrm{t}_{1}=13.86 \mathrm{~min}$ (minor), $\mathrm{t}_{2}=16.60$ (major).

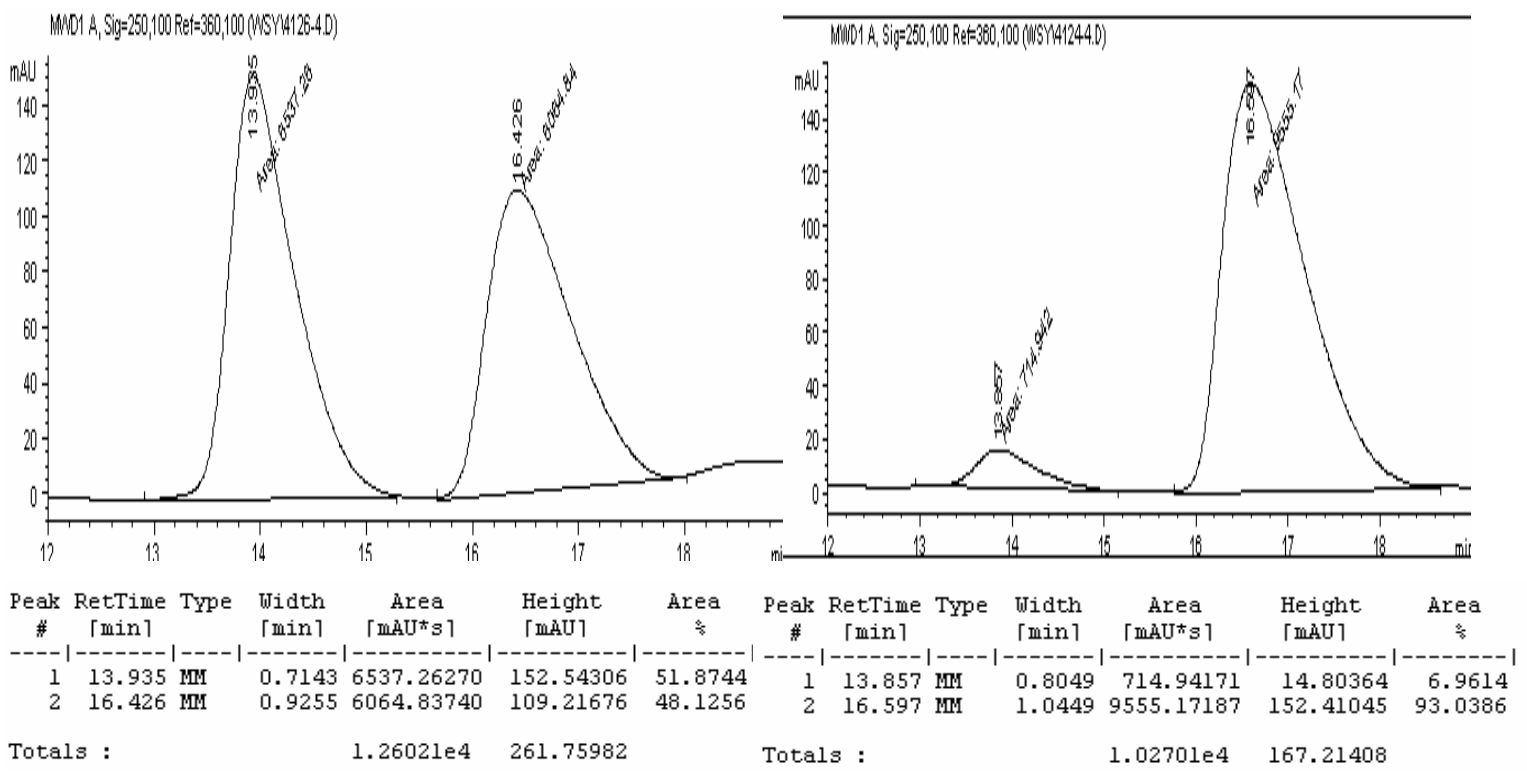




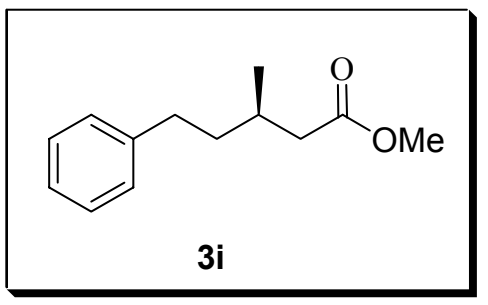

(+)-Methyl 3-methyl-5-phenylpentanoate

(Table 2, entry 9): colorless oil, $20 \%$ yield, $99 \%$ ee, $[\alpha]_{\mathrm{D}}{ }^{20}=+7.5(c=1.7)$;

FTIR (KBr, neat): $v 1737(\mathrm{C}=\mathrm{O})$;

${ }^{1} \mathbf{H}$ NMR (300 MHz, $\left.\mathbf{C D C l}_{3}\right): \delta$ 7.18-7.30 (m, 3H), $3.66(\mathrm{~s}, 3 \mathrm{H}), 2.53-2.72(\mathrm{~m}, 2 \mathrm{H}), 2.36(\mathrm{dd}, J=14.7$ and 6.1 $\mathrm{Hz}, 1 \mathrm{H}), 2.20(\mathrm{dd}, J=14.7$ and $8.0 \mathrm{~Hz}), 1.97(\mathrm{~m}, 1 \mathrm{H}), 1.72-1.45(\mathrm{~m}, 2 \mathrm{H}), 1.01(\mathrm{~d}, J=6.6 \mathrm{~Hz}, 3 \mathrm{H})$;

${ }^{13}$ C NMR (75.4 MHz, $\left.\mathbf{C D C l}_{3}\right): \delta 173.5(\mathrm{C}), 142.3(\mathrm{C}), 128.3(\mathrm{CH}), 128.3(\mathrm{CH}), 125.7(\mathrm{CH}), 51.4\left(\mathrm{CH}_{3}\right), 41.5$ $\left(\mathrm{CH}_{2}\right), 38.5\left(\mathrm{CH}_{2}\right), 33.3(\mathrm{CH} 2), 30.1(\mathrm{CH}), 19.6\left(\mathrm{CH}_{3}\right)$;

HRMS calcd. For $\mathrm{C}_{13} \mathrm{H}_{18} \mathrm{O}_{2}$ 206.1307, found 206.1292;

The enantiomeric excess was determined by chiral HPLC analysis, Chiralcel OD-H (Hexane : $i$-propanol 99.2 : $0.8,1 \mathrm{~mL} / \mathrm{min}$ ): $\mathrm{t}_{1}=6.80 \mathrm{~min}$ (minor), $\mathrm{t}_{2}=7.58$ (major).

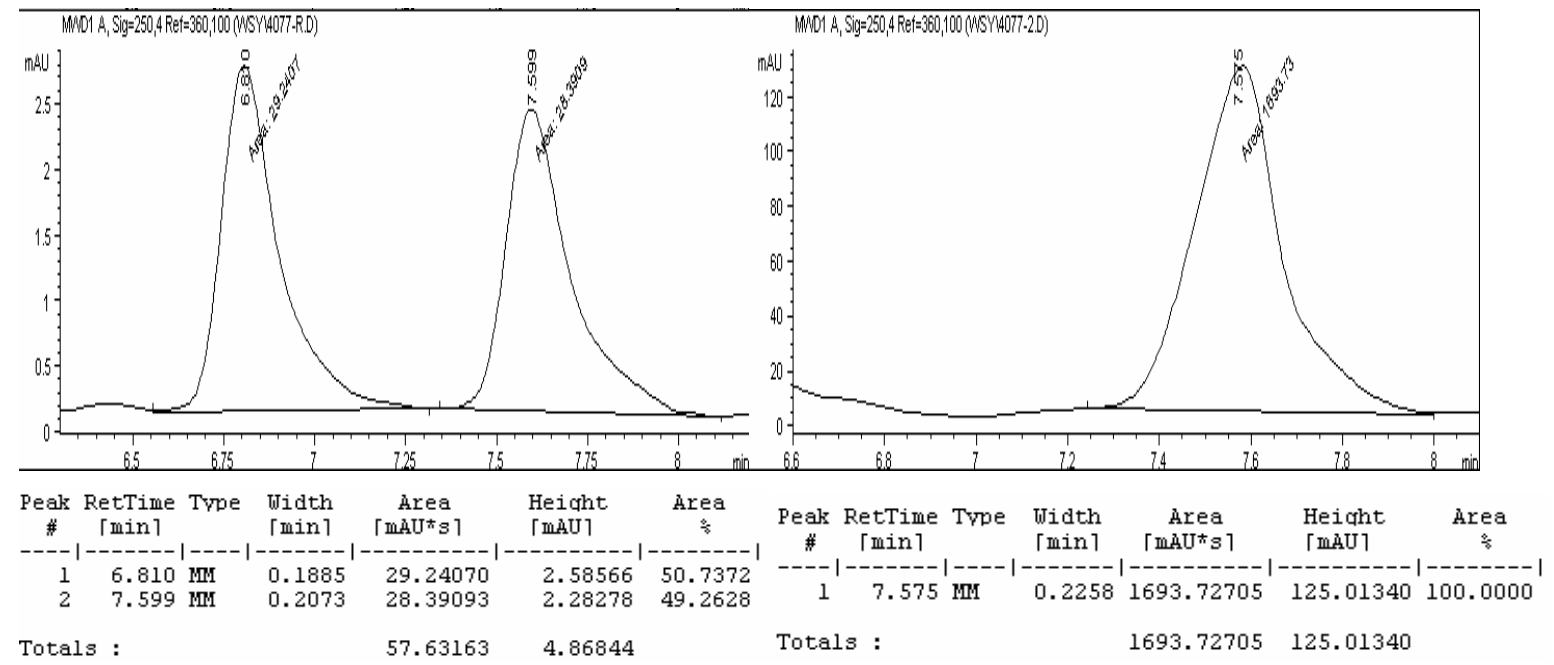




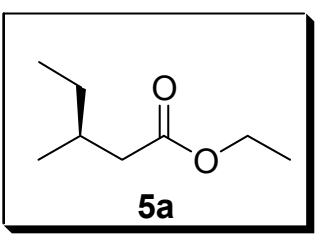

(S)-(+)-Ethyl 3-methylpentanoate

(Table 3, entry 1): colorless oil, $83 \%$ yield, $74 \%$ ee, $[\alpha]_{\mathrm{D}}{ }^{25}=+7.2(c=0.6)$, lit. $^{2}$ for $(\mathrm{S})$; enantiomer $[\alpha]_{\mathrm{D}}{ }^{25}=$ $+10.0(c=0.6)$;

FTIR (KBr, neat): $v 1732(\mathrm{C}=\mathrm{O})$;

${ }^{1}$ H NMR (300 MHz, CDCl $): \delta 4.09$ (q, $\left.J=7.1 \mathrm{~Hz}, 2 \mathrm{H}\right), 2.26(\mathrm{dd}, J=14.6$ and $6.1 \mathrm{~Hz}, 1 \mathrm{H}), 2.05$ (dd, $J=14.6$ and $8.1 \mathrm{~Hz}, 1 \mathrm{H}), 1.79-1.90(\mathrm{~m}, 1 \mathrm{H}), 1.29-1.38(\mathrm{~m}, 2 \mathrm{H}), 1.22(\mathrm{t}, J=7.1 \mathrm{~Hz}, 3 \mathrm{H}), 0.83-0.90(\mathrm{~m}, 6 \mathrm{H})$;

${ }^{13}$ C NMR (75.4 MHz, $\left.\mathbf{C D C l}_{3}\right): \delta$ 173.4, 60.0, 41.5, 31.9, 29.3, 19.2, 14.2, 11.2;

HRMS calcd. For $\mathrm{C}_{8} \mathrm{H}_{16} \mathrm{O}_{2}$ 144.1150, found 144.1155;

The enantiomeric excess determined by chiral GC analysis, Chiraldex G-TA column $(30 \mathrm{~m} \times 0.25 \mathrm{~mm}), 60^{\circ} \mathrm{C}$, retention times $(\mathrm{min}): \mathrm{t}_{1}=16.29 \min \left(\right.$ minor), $\mathrm{t}_{2}=18.25 \mathrm{~min}$ (major).

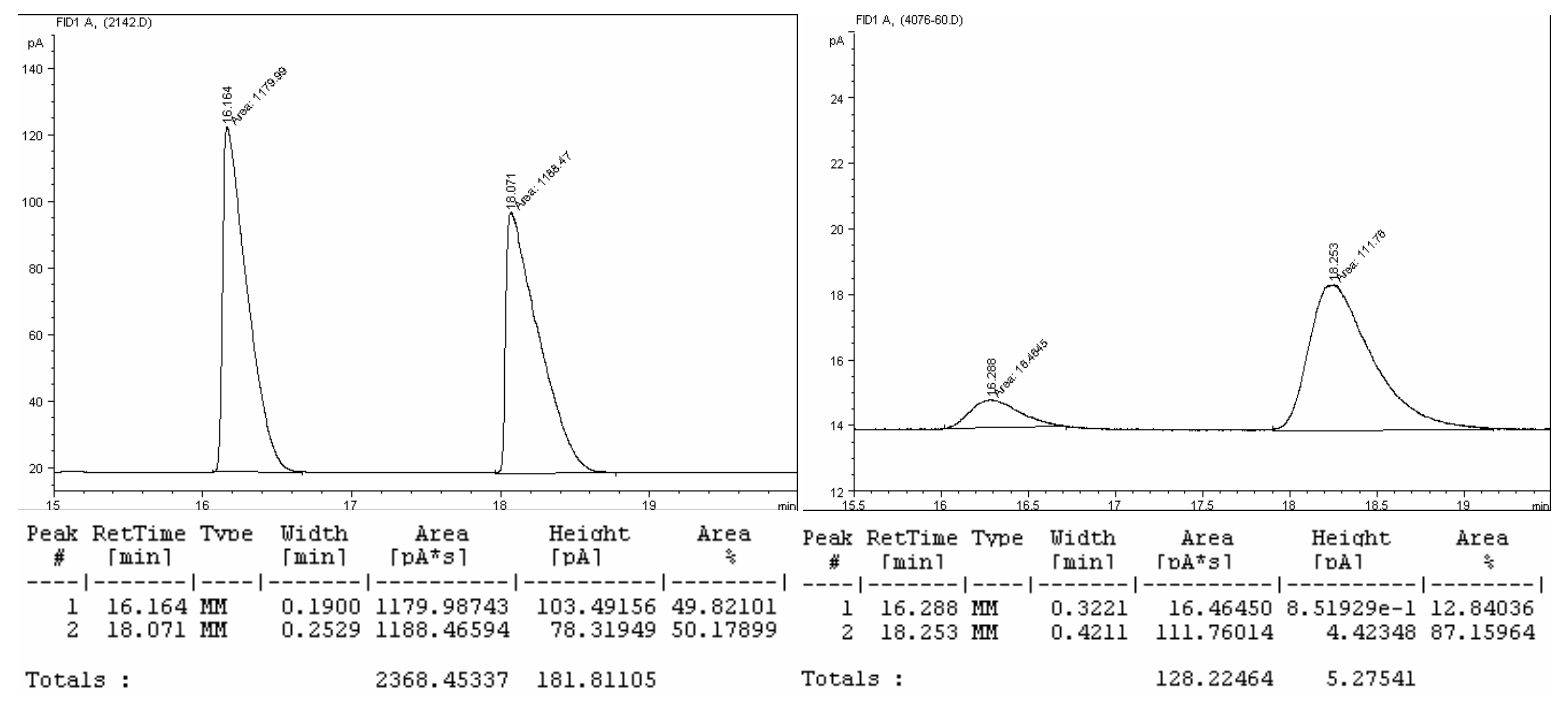




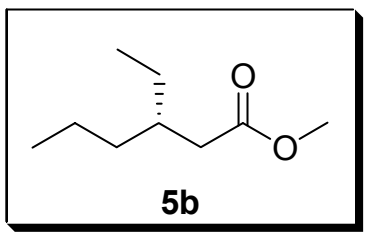

(S)-(-)-Methyl 3-ethylhexanoate

(Table 3, entry 2): colorless oil, $85 \%$ yield, $87 \%$ ee, $[\alpha]_{\mathrm{D}}{ }^{25}=-2.6(c=1.3)$, lit. $^{3}$ for $(\mathrm{S})$ enantiomer: $[\alpha]_{\mathrm{D}}{ }^{25}=-2.1$;

FTIR (KBr, neat): $v 1732(\mathrm{C}=\mathrm{O})$;

${ }^{1}$ H NMR (400 MHz, CDCl $): \delta 3.64(\mathrm{~s}, 3 \mathrm{H}), 2.22(\mathrm{~d}, J=7.2 \mathrm{~Hz}, 2 \mathrm{H}), 1.76-1.83(\mathrm{~m}, 1 \mathrm{H}), 1.20-1.41(\mathrm{~m}, 6 \mathrm{H})$, 0.80-0.95 (m, 6H);

${ }^{13} \mathbf{C}$ NMR (100.0 MHz, $\left.\mathbf{C D C l}_{3}\right): \delta$ 174.1 (C), $51.3\left(\mathrm{CH}_{3}\right), 38.6\left(\mathrm{CH}_{2}\right), 36.2(\mathrm{CH}), 35.7\left(\mathrm{CH}_{2}\right), 26.3\left(\mathrm{CH}_{2}\right), 19.7$ $\left(\mathrm{CH}_{2}\right), 14.3\left(\mathrm{CH}_{3}\right), 10.7\left(\mathrm{CH}_{3}\right)$;

HRMS calcd. For $\mathrm{C}_{9} \mathrm{H}_{18} \mathrm{O}_{2}$ 158.1307, found 158.1301;

The enantiomeric excess was determined by chiral GC analysis, Chiraldex G-TA column (30 m x $0.25 \mathrm{~mm}$ ), $60^{\circ} \mathrm{C}$, retention times $(\mathrm{min}): \mathrm{t}_{1}=34.67$ min (major), $\mathrm{t}_{2}=36.30$ (minor).

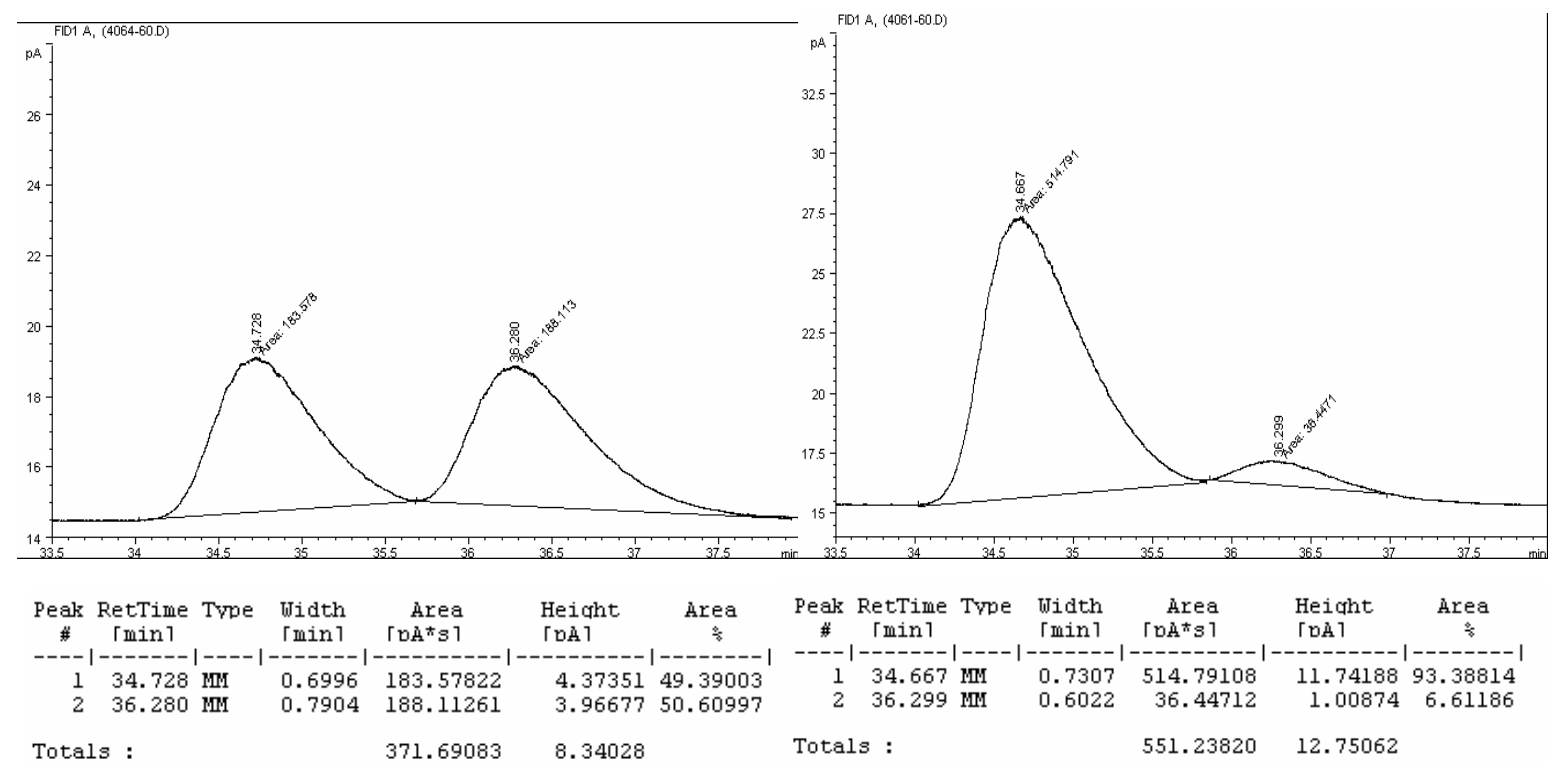

3. Schuppan, J.; Minnaard, A. J.; Feringa, B. L. Chem. Commun. 2004, 7, 792-793. 


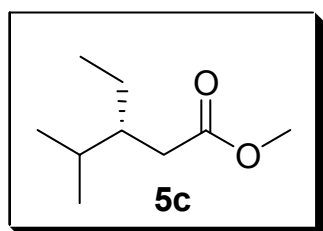

(R)-(-)-Methyl 3-ethyl-4-methylpentanoate

(Table 3, entry 3): colorless oil, $90 \%$ yield, $95 \%$ ee, colorless oil, [a $]_{\mathrm{D}}{ }^{25}=-3.8(c=1.4)$, lit. $^{2}$ for $(S)$ enantiomer: $[\alpha]_{\mathrm{D}}^{25}=+4.4$.

FTIR (KBr, neat): $v 1732(\mathrm{C}=\mathrm{O})$;

${ }^{1}$ H NMR (300 MHz, $\mathbf{C D C l}_{3}$ ): $\delta 3.66(\mathrm{~s}, 3 \mathrm{H}), 2.28(\mathrm{dd}, J=15.1$ and $5.9 \mathrm{~Hz}, 1 \mathrm{H}), 2.15(\mathrm{dd}, J=15.1$ and $7.4 \mathrm{~Hz}$, $1 \mathrm{H}), 1.66-1.78(\mathrm{~m}, 2 \mathrm{H}), 1.17-1.44(\mathrm{~m}, 2 \mathrm{H}), 0.80-0.90(\mathrm{~m}, 9 \mathrm{H})$;

${ }^{13}$ C NMR (75.4 MHz, $\left.\mathbf{C D C l}_{3}\right): \delta$ 174.7, 51.4, 42.5, 35.6, 29.4, 23.7, 19.4, 18.5, 11.7 .

HRMS calcd. For $\mathrm{C}_{9} \mathrm{H}_{18} \mathrm{O}_{2}$ 158.1307, found 158.1301;

The enantiomeric excess determined by chiral GC analysis, Chiraldex G-TA column $(30 \mathrm{~m} \times 0.25 \mathrm{~mm}), 60^{\circ} \mathrm{C}$, retention times $(\mathrm{min}): \mathrm{t}_{1}=31.80 \min$ (major), $\mathrm{t}_{2}=33.87$ (minor).

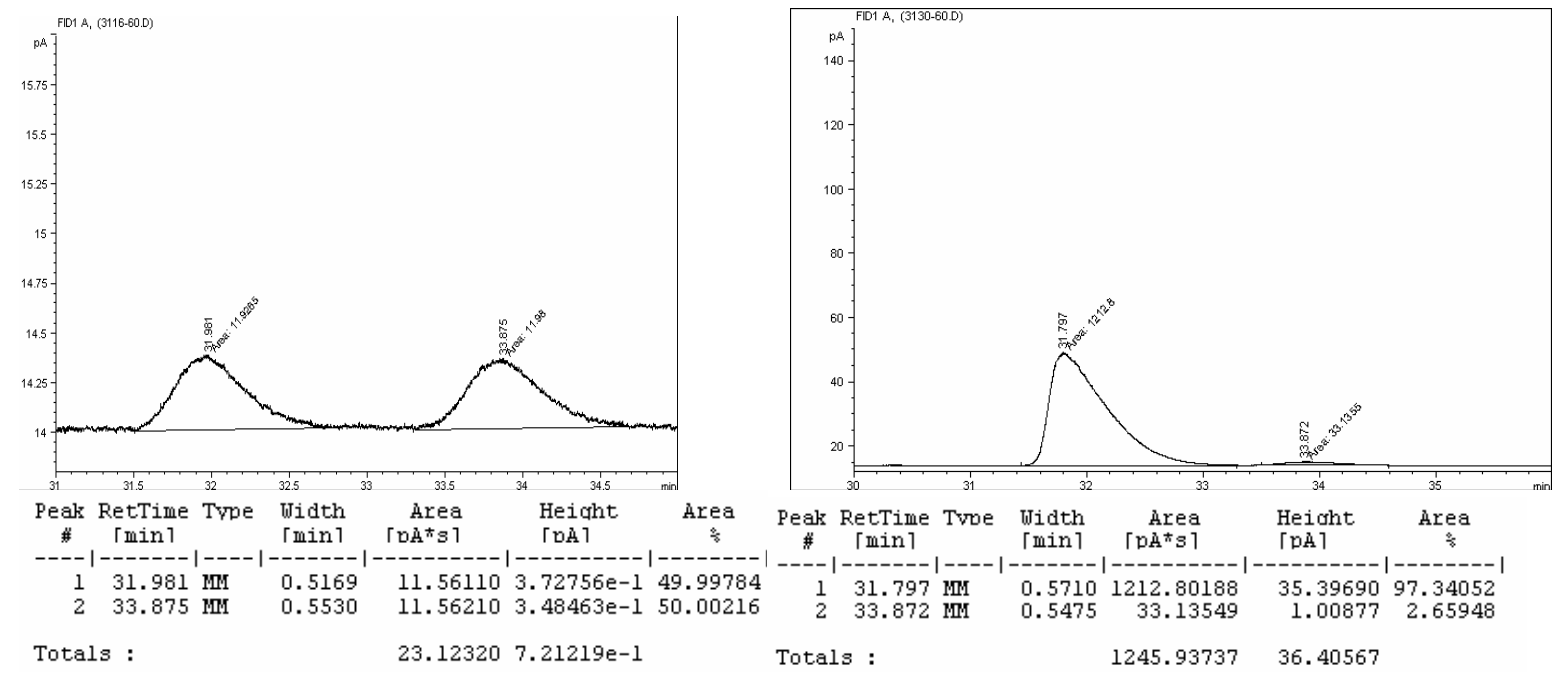




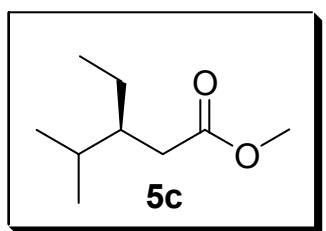

\section{(S)-(+)-Methyl 3-ethyl-4-methylpentanoate}

colorless oil, $75 \%$ yield, $56 \%$ ee, colorless oil, $[\alpha]_{\mathrm{D}}^{25}=+3.9(c=1.8)$, lit. for $(S)^{2}$ enantiomer: $[\mathrm{a}]_{\mathrm{D}}{ }^{25}=+4.4$.

The enantiomeric excess determined by chiral GC analysis, Chiraldex G-TA column (30 m x $0.25 \mathrm{~mm}), 60^{\circ} \mathrm{C}$, retention times ( $\mathrm{min}$ ): $\mathrm{t}_{1}=31.69 \min$ (major), $\mathrm{t}_{2}=33.32$ (minor).

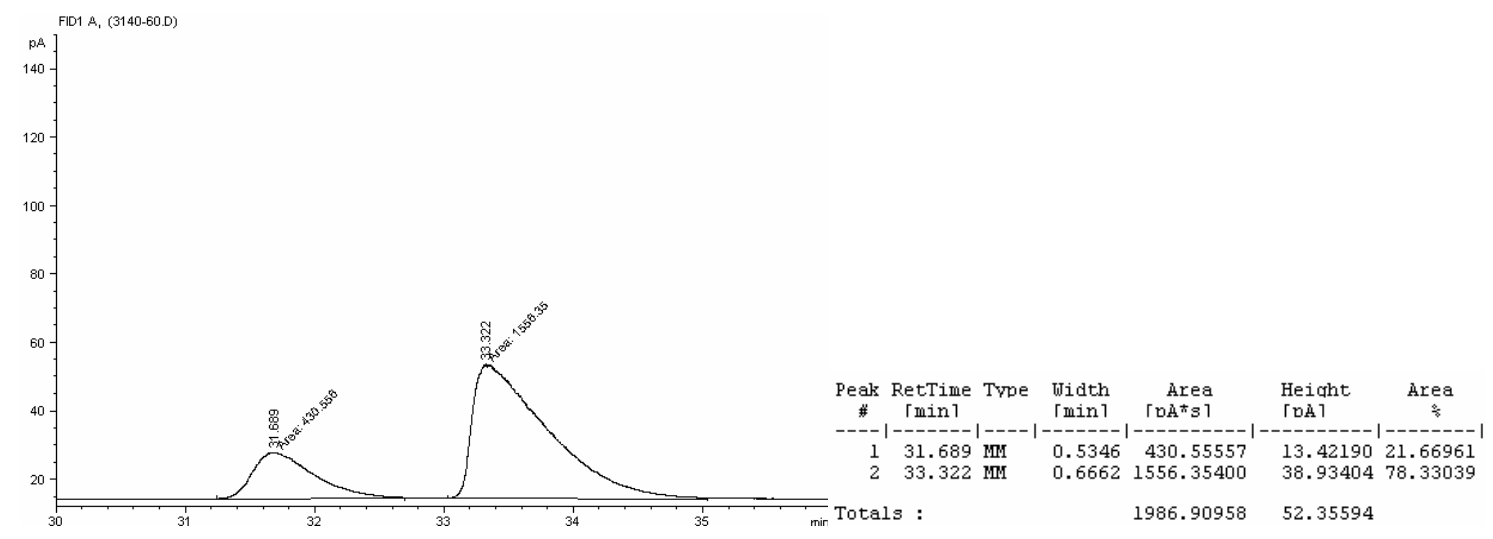




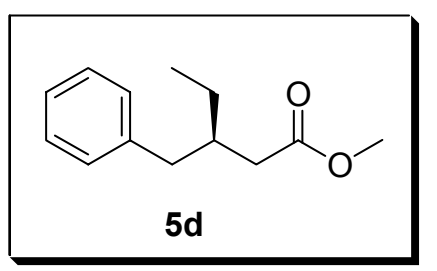

(-)-Methyl 3-hydroxy-2,2-dimethyl-5-phenylpentanoate $\mathbf{R}_{\boldsymbol{f}}=0.70(\mathrm{EA} / \mathrm{Hexane}=1 / 4)$

(Table 3, entry 7): colorless oil, $85 \%$ yield, $94 \%$ ee, colorless oil, $[\alpha]_{\mathrm{D}}{ }^{25}=-5.9(c=1.6)$.

FTIR (KBr, neat): $v 1735(\mathrm{C}=\mathrm{O})$;

${ }^{1}$ H NMR (400 MHz, $\left.\mathbf{C D C l}_{3}\right): \delta$ 7.17-7.30 (m, 5H), $3.63\left(\mathrm{~s}, 3 \mathrm{H}, \mathrm{OCH}_{3}\right), 2.67(\mathrm{dd}, J=13.6$ and $6.8 \mathrm{~Hz}, 1 \mathrm{H}), 2.54$ $(\mathrm{dd}, J=13.5$ and $5.5 \mathrm{~Hz}), 2.14-2.17(\mathrm{~m}, 2 \mathrm{H}), 2.10-2.12(\mathrm{~m}, 1 \mathrm{H}), 1.22-1.42(\mathrm{~m}, 2 \mathrm{H}), 0.95(\mathrm{t}, J=6.8 \mathrm{~Hz}, 3 \mathrm{H})$;

${ }^{13}$ C NMR (100.0 MHz, $\left.\mathbf{C D C l}_{3}\right): \delta 173.8(\mathrm{C}), 140.1(\mathrm{C}), 129.3(\mathrm{CH}), 128.3(\mathrm{CH}), 126.0(\mathrm{CH}), 51.4\left(\mathrm{CH}_{3}\right), 40.0$ $\left(\mathrm{CH}_{2}\right), 38.5(\mathrm{CH}), 37.9\left(\mathrm{CH}_{2}\right), 26.2\left(\mathrm{CH}_{2}\right), 11.0\left(\mathrm{CH}_{3}\right)$;

HRMS calcd. For $\mathrm{C}_{13} \mathrm{H}_{18} \mathrm{O}_{2} 206.1307$, found 158.1296;

The enantiomeric excess was determined by HPLC analysis employing a Daicel Chiracel ODH column (Hexane : $i$-propanol $99.2: 0.8,1 \mathrm{~mL} / \mathrm{min}$ ): $\mathrm{t}_{1}=6.23 \mathrm{~min}$ (minor), $\mathrm{t}_{2}=7.79$ (major).

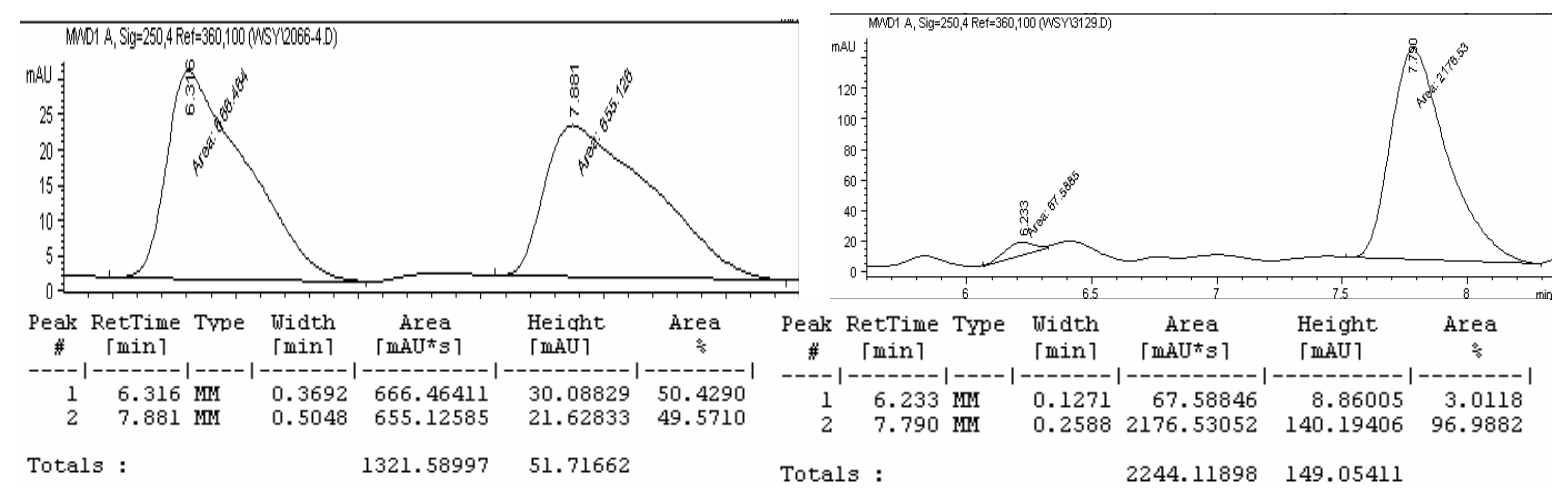




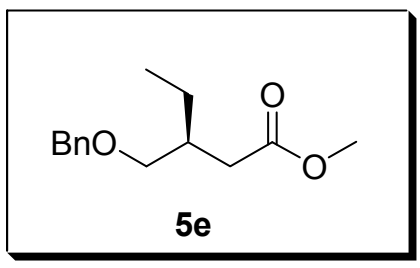

(-)-Methyl 3-((benzyloxy)methyl)pentanoate

(Table 3, entry 8): colorless oil, $83 \%$ yield, $73 \%$ ee, $[\alpha]_{\mathrm{D}}{ }^{25}=-6.0(c=1.0)$;

FTIR (KBr, neat): $v 1732(\mathrm{C}=\mathrm{O})$;

${ }^{1}$ H NMR (300 MHz, $\left.\mathbf{C D C l}_{3}\right): \delta 7.32(\mathrm{~m}, 5 \mathrm{H}), 4.49(\mathrm{~s}, 2 \mathrm{H}), 3.63(\mathrm{~s}, 3 \mathrm{H}), 3.45(\mathrm{dd}, J=9.3$ and $5.0 \mathrm{~Hz}, 1 \mathrm{H}), 3.35$ $(\mathrm{dd}, J=9.2$ and $6.7 \mathrm{~Hz}, 1 \mathrm{H}), 2.43(\mathrm{dd}, J=15.4$ and $7.3 \mathrm{~Hz}, 1 \mathrm{H}), 2.30(\mathrm{dd}, J=15.3$ and $6.3 \mathrm{~Hz}, 1 \mathrm{H}), 2.08-2.14$ (m, 1H), 1.23-1.48 (m, 2H), $0.90(\mathrm{t}, J=7.5 \mathrm{~Hz}, 3 \mathrm{H})$;

${ }^{13}$ C NMR (75.4 MHz, $\left.\mathbf{C D C l}_{3}\right): \delta 173.8(\mathrm{C}), 138.6(\mathrm{C}), 128.3(\mathrm{CH}), 127.5(\mathrm{CH}), 127.4(\mathrm{CH}), 73.0\left(\mathrm{CH}_{2}\right), 72.5$ $\left(\mathrm{CH}_{2}\right), 51.4\left(\mathrm{CH}_{3}\right), 37.3(\mathrm{CH}), 36.5\left(\mathrm{CH}_{2}\right), 24.2\left(\mathrm{CH}_{2}\right), 11.2\left(\mathrm{CH}_{3}\right)$;

HRMS calcd. For $\mathrm{C}_{14} \mathrm{H}_{20} \mathrm{O}_{3}$ 236.1412, found 236.1398;

The enantiomeric excess was determined by HPLC analysis employing a Daicel Chiracel ODH column (Hexane : $i$-propanol $99.2: 0.8,1 \mathrm{~mL} / \mathrm{min}$ ): $\mathrm{t}_{1}=7.65 \mathrm{~min}$ (minor), $\mathrm{t}_{2}=8.21$ (major).

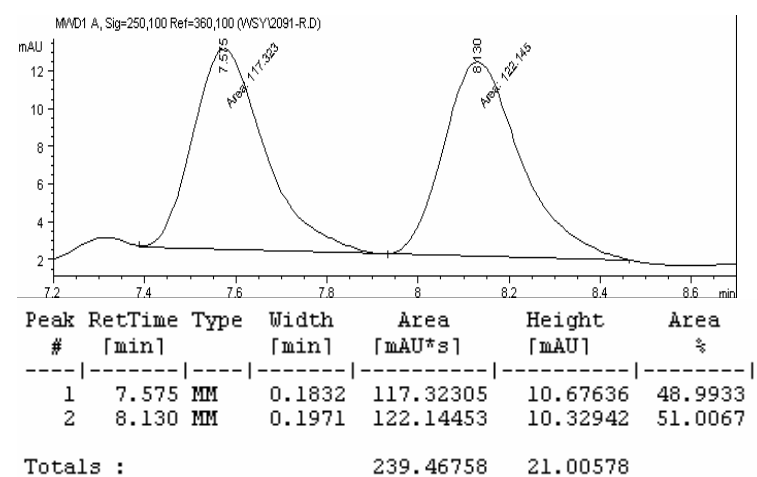

Totals :

239.46758

21.00578

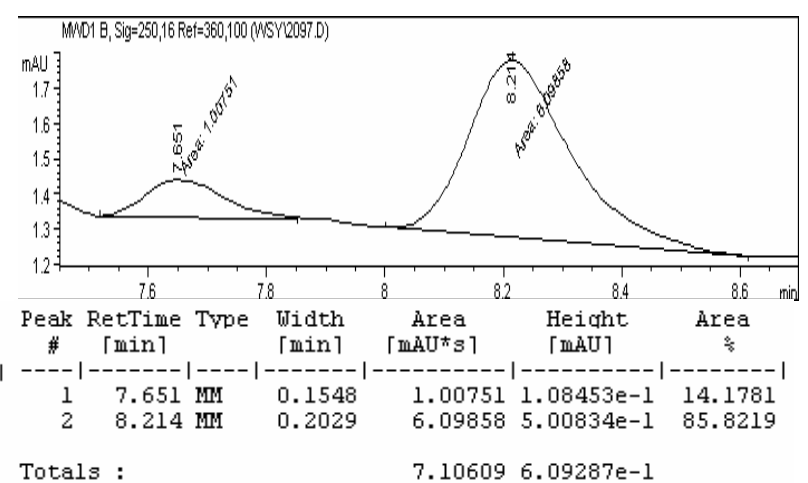




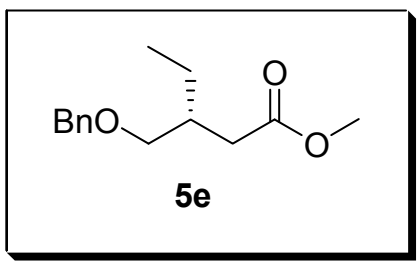

\section{(+)-Methyl 3-((benzyloxy)methyl)pentanoate}

(Table 3, entry 9): colorless oil, 86\% yield, $87 \%$ ee, $[\alpha]_{D}^{25}=+6.2(c=0.5)$;

The enantiomeric excess was determined by HPLC analysis employing a Daicel Chiracel ODH column (Hexane : $i$-propanol $99.2: 0.8,1 \mathrm{~mL} / \mathrm{min}$ ): $\mathrm{t}_{1}=7.95 \min$ (major), $\mathrm{t}_{2}=8.49$ (minor).

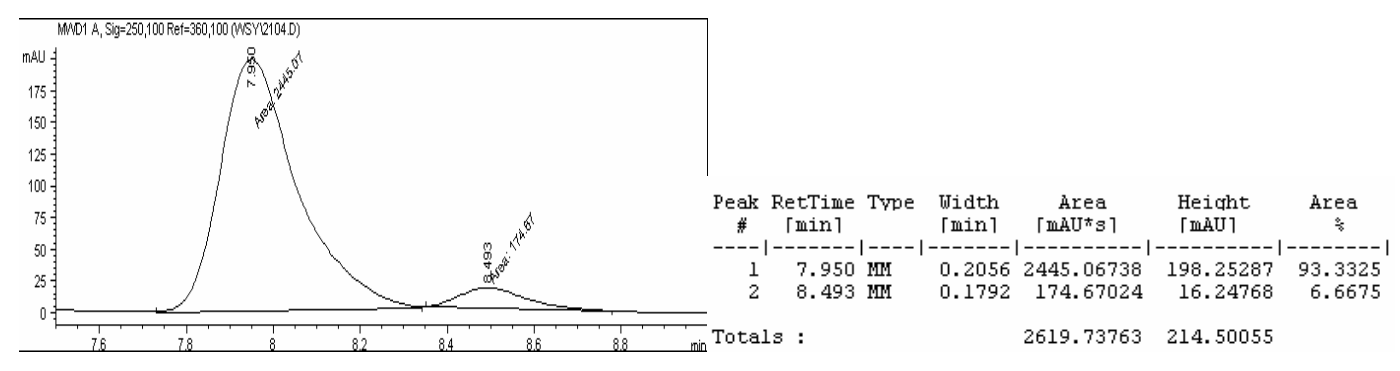




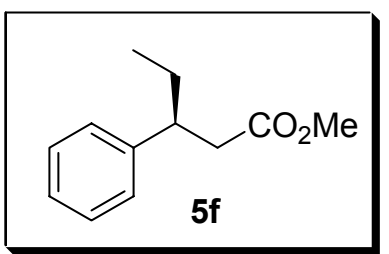

(S)-(+)-Methyl 3-phenylpentanoate

(Table 3, entry 10): $90 \%$ yield, $93 \%$ ee, colorless oil, $[\alpha]_{\mathrm{D}}{ }^{25}=+15.5(c=0.4)$, lit. ${ }^{2}$ for : $(S)$ enantiomer: $[\alpha]_{\mathrm{D}}{ }^{25}=$ +18.7 .

FTIR (KBr, neat): $v 1737 \mathrm{~cm}^{-1}$

${ }^{1}$ H NMR (300 MHz, CDCl $): \delta$ 7.09-7.22 (m, 5H), $3.51(\mathrm{~s}, 3 \mathrm{H}), 2.88-2.98(\mathrm{~m}, 1 \mathrm{H}), 2.46-2.63(\mathrm{~m}, 2 \mathrm{H}), 1.51-$ $1.68(\mathrm{~m}, 2 \mathrm{H}), 0.75(\mathrm{t}, J=7.4 \mathrm{~Hz}, 3 \mathrm{H})$;

${ }^{13}$ C NMR (75.4 MHz, $\left.\mathbf{C D C l}_{3}\right): \delta 173.0(\mathrm{C}), 143.9(\mathrm{C}), 128.4(\mathrm{CH}), 127.5(\mathrm{CH}), 126.4(\mathrm{CH}), 51.4\left(\mathrm{CH}_{3}\right), 43.8$ (CH), $41.3\left(\mathrm{CH}_{2}\right), 29.1\left(\mathrm{CH}_{2}\right), 11.9\left(\mathrm{CH}_{3}\right)$;

HRMS calcd. For $\mathrm{C}_{12} \mathrm{H}_{16} \mathrm{O}_{2}$ 192.1150, found 192.1136;

The enantiomeric excess was determined by chiral GC analysis, Chiraldex G-TA column (30 m x $0.25 \mathrm{~mm}$ ), $90^{\circ} \mathrm{C}, \mathrm{t}_{1}=72.00 \mathrm{~min}$ (minor), $\mathrm{t}_{2}=74.65$ (major).

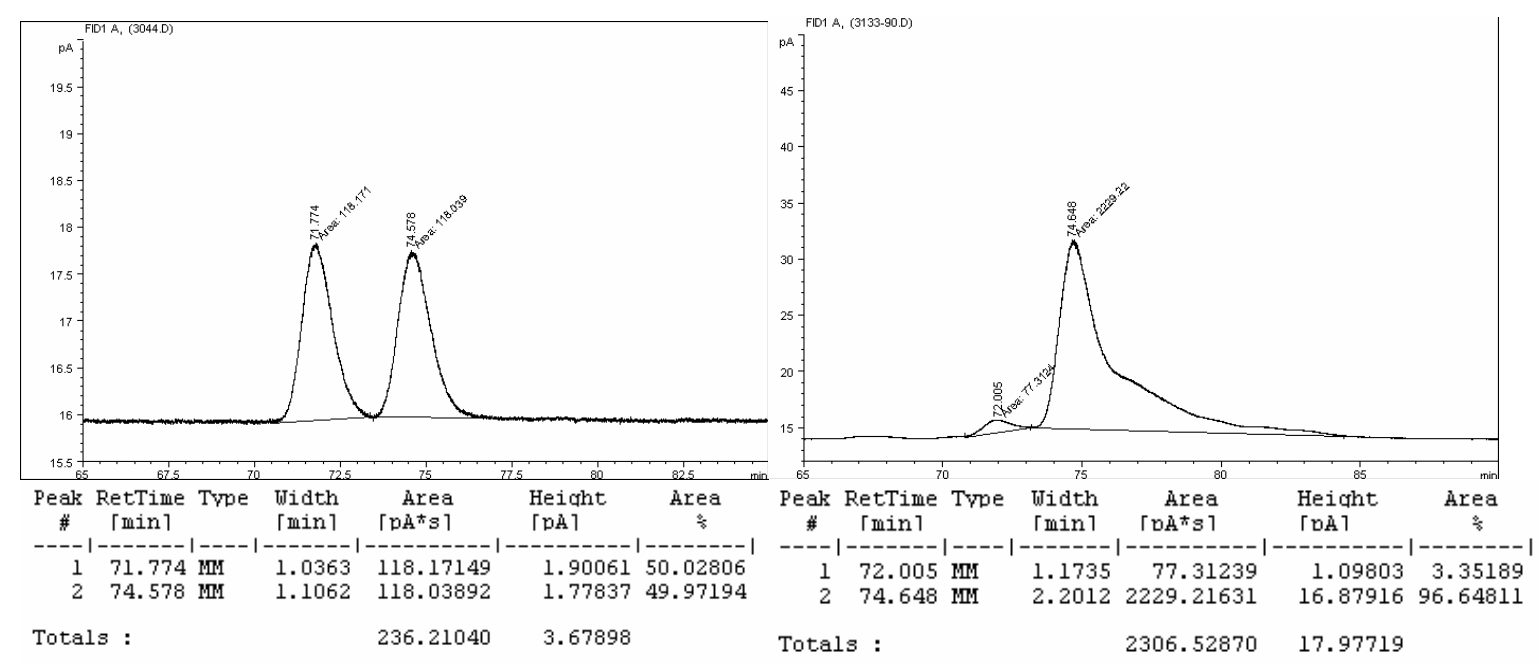




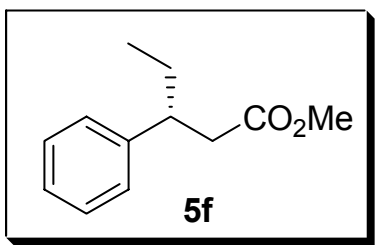

(R)-(-)-Methyl 3-phenylpentanoate

$80 \%$ yield, $74 \%$ ee, colorless oil, $[\alpha]_{\mathrm{D}}{ }^{25}=+15.5(c=0.4)$

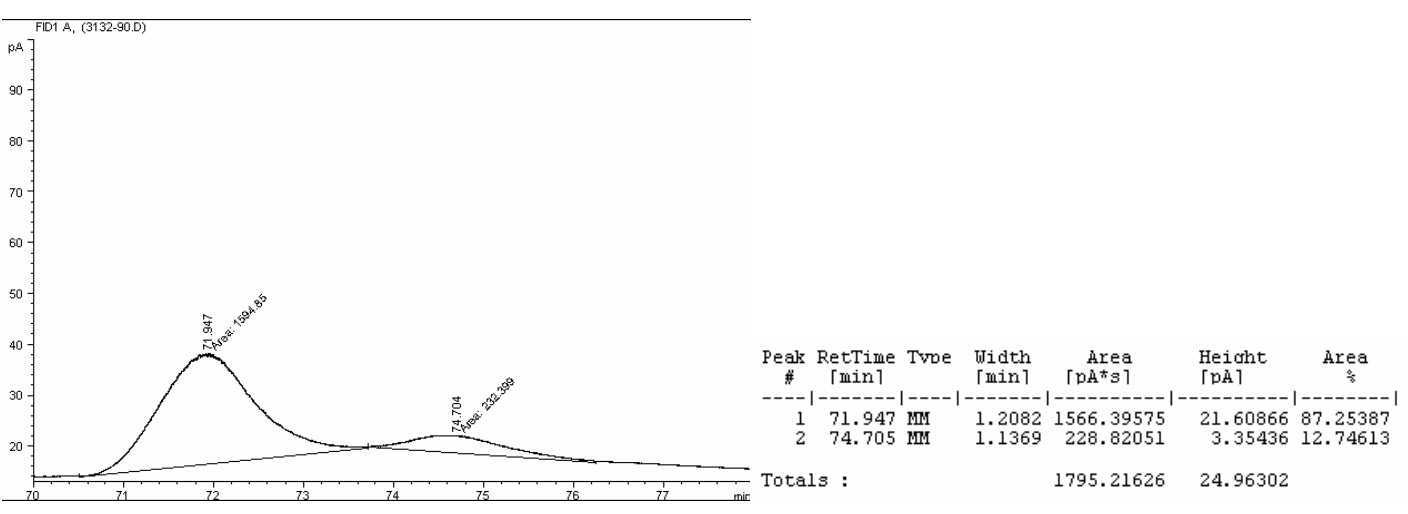




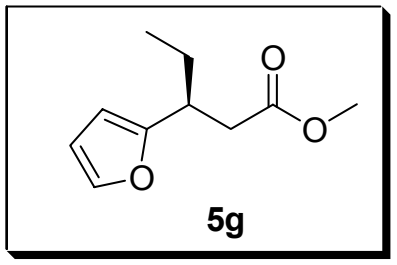

(+)-Methyl 3-(furan-2-yl)pentanoate

(Table 3, entry 12): colorless oil, $80 \%$ yield, $85 \%$ ee, $[\alpha]_{\mathrm{D}}{ }^{20}=+14.2(c=0.4)$, lit. $^{2}[\alpha]_{\mathrm{D}}{ }^{20}=+1.7$;

FTIR (KBr, neat): $v 1737(\mathrm{C}=\mathrm{O})$;

${ }^{1}$ H NMR (300 MHz, CDCl$): \delta 7.31(\mathrm{~s}, 1 \mathrm{H}), 6.27(\mathrm{~m}, 1 \mathrm{H}), 6.03(\mathrm{~d}, J=3.0 \mathrm{~Hz}, 1 \mathrm{H}), 3.64(\mathrm{~s}, 3 \mathrm{H}), 3.12-3.21(\mathrm{~m}$, $1 \mathrm{H}), 2.53-2.69(\mathrm{~m}, 2 \mathrm{H}), 1.60-1.72(\mathrm{~m}, 2 \mathrm{H}), 0.83(\mathrm{t}, J=7.3 \mathrm{~Hz}, 3 \mathrm{H})$;

${ }^{13}$ C NMR (75.4 MHz, $\left.\mathbf{C D C l}_{3}\right): \delta 172.7(\mathrm{C}), 157.08(\mathrm{C}), 141.1(\mathrm{CH}), 109.9(\mathrm{CH}), 105.2(\mathrm{CH}), 51.6\left(\mathrm{CH}_{3}\right), 38.4$ $\left(\mathrm{CH}_{2}\right), 37.0(\mathrm{CH}), 26.7\left(\mathrm{CH}_{2}\right), 11.4\left(\mathrm{CH}_{3}\right)$;

HRMS calcd. For $\mathrm{C}_{10} \mathrm{H}_{14} \mathrm{O}_{3}$ 182.0943, found 182.0937;

The enantiomeric excess was determined by chiral GC analysis, Chiraldex GTA column $(30 \mathrm{~m} \times 0.25 \mathrm{~mm}), 90^{\circ} \mathrm{C}$, retention times $(\mathrm{min})$ : $\mathrm{t}_{1}=26.01 \mathrm{~min}$ (minor), $\mathrm{t}_{2}=26.96$ (major).
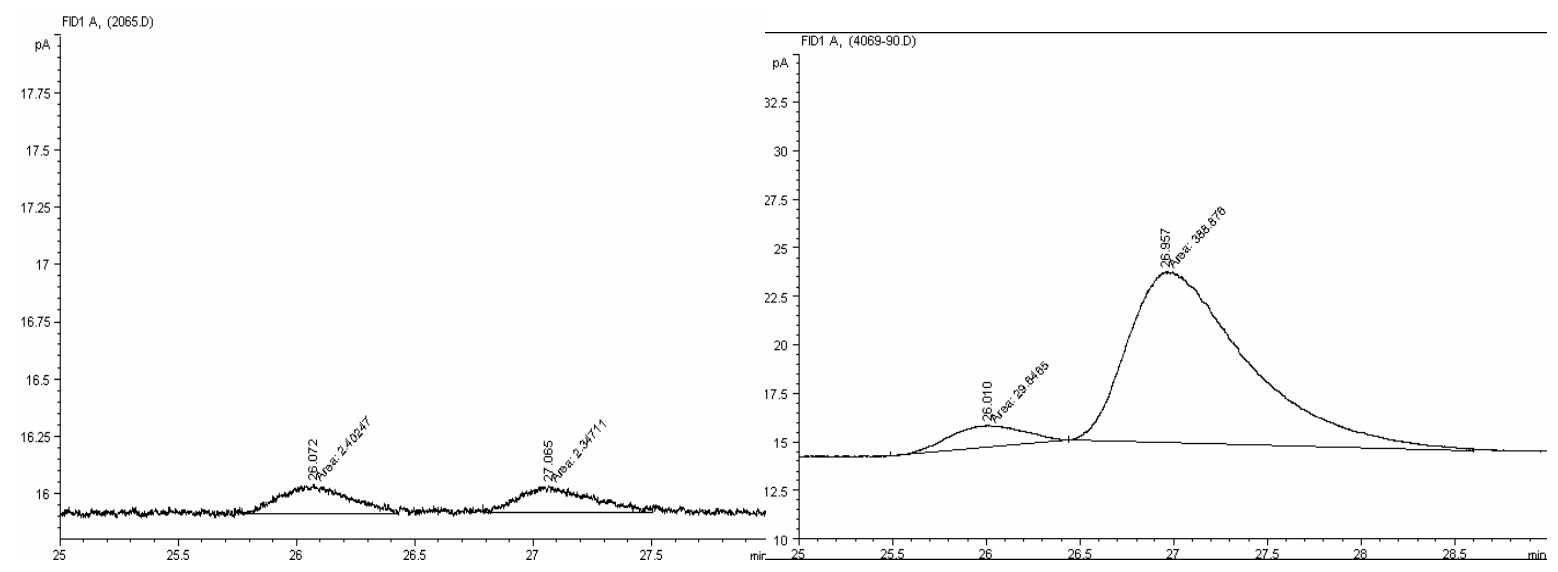

\begin{tabular}{|c|c|c|c|c|c|c|c|c|c|c|c|}
\hline $\begin{array}{c}\text { Peak } \\
\#\end{array}$ & $\begin{array}{l}\text { RetTime Type } \\
\text { 「min } 1\end{array}$ & $\begin{array}{l}\text { Width } \\
\text { 「min1 }\end{array}$ & $\begin{array}{r}\text { Area } \\
\text { [0A*s }\end{array}$ & $\begin{array}{l}\text { Height } \\
\text { 「DA } 1\end{array}$ & $\underset{\substack{\text { Area } \\
\$}}{*}$ & $\begin{array}{c}\text { Peak } \\
\#\end{array}$ & $\begin{array}{l}\text { RetTime Tvoe } \\
\text { 「min }\end{array}$ & $\begin{array}{l}\text { Width } \\
\text { 「min1 }\end{array}$ & $\begin{array}{r}\text { Area } \\
\left\lceil\not A^{*} S\right\rceil\end{array}$ & $\begin{array}{l}\text { Heioht } \\
\text { 「pA }\rceil\end{array}$ & $\underset{\$}{\text { Area }}$ \\
\hline$\frac{1}{2}$ & $\begin{array}{l}26.072 \mathrm{MM} \\
27.065 \mathrm{MM}\end{array}$ & $\begin{array}{l}0.3103 \\
0.3360\end{array}$ & $\begin{array}{l}2.40247 \\
2.34711\end{array}$ & $\begin{array}{l}1.29050 \mathrm{e}-1 \\
1.16435 \mathrm{e}-1\end{array}$ & $\begin{array}{l}50.58284 \\
49.41716\end{array}$ & $\frac{1}{2}$ & $\begin{array}{l}26.010 \mathrm{MM} \\
26.957 \mathrm{mM}\end{array}$ & $\begin{array}{l}0.4530 \\
0.7352\end{array}$ & $\begin{array}{r}29.64649 \\
388.87567\end{array}$ & $\begin{array}{l}1.09083 \\
8.81612\end{array}$ & $\begin{array}{r}7.08361 \\
92.91639\end{array}$ \\
\hline Total & ls: & & 4.74958 & $2.45485 e-1$ & & Tota & ls : & & 418.52216 & 9.90695 & \\
\hline
\end{tabular}


Figure 2 The crystal-structure of $\mathrm{Cu}$ dimer complex 1a

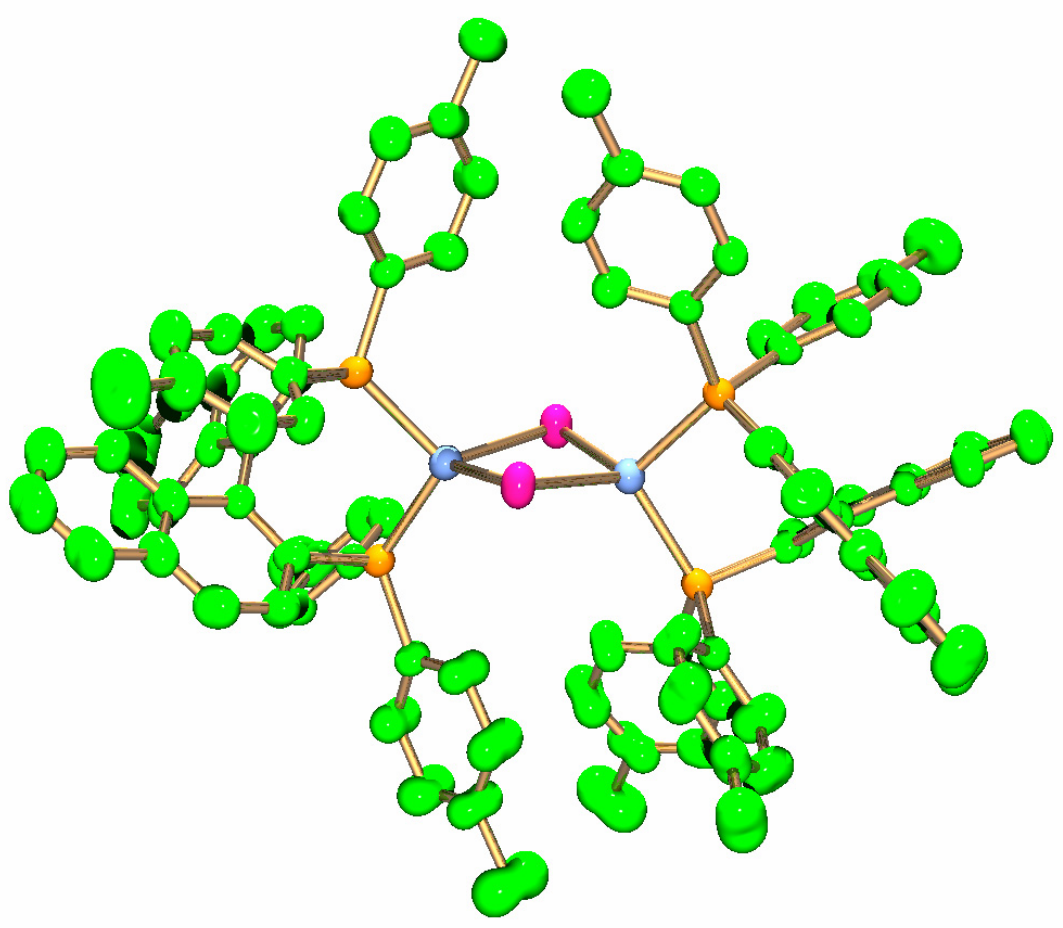

Figure 3 The spacefill of $\mathrm{Cu}$ dimer complex 1a

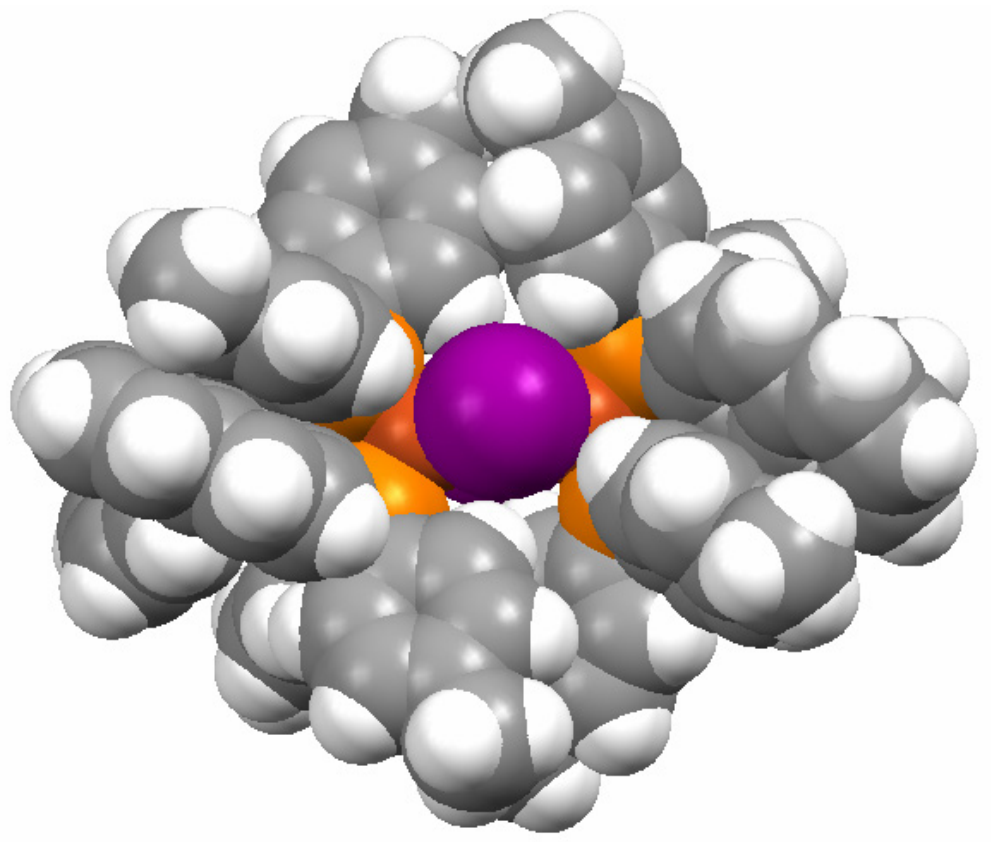

